\title{
Radial profiles of Fe abundance in the intracluster medium of nearby clusters observed with XMM-Newton
}

\author{
K. Matsushita \\ Department of Physics, Tokyo University of Science, 1-3 Kagurazaka, Shinjyuku-ku, Tokyo, 162-8601, Japan \\ e-mail: matusita@rs.kagu.tus.ac.jp
}

Received 9 October 2009 / Accepted 5 January 2011

\section{ABSTRACT}

\begin{abstract}
Aims. The abundances of $\mathrm{Fe}$ in the intracluster medium of nearby $(z<0.08)$ clusters were measured up to $0.3 \sim 0.5 r_{180}$. Methods. We analyzed 28 clusters of galaxies observed with XMM-Newton. We derived Fe abundances from the flux ratios of Fe lines to the continuum within an energy range of 3.5-6 keV to minimize and evaluate systematic uncertainties.

Results. The radial profiles of the Fe abundances of relaxed clusters with a cD galaxy at their X-ray peak have similar slopes. These clusters show similar enhancements in the Fe abundance within $0.1 r_{180}$, and at $0.1-0.3 r_{180}$, they have flatter Fe abundance profiles at $0.4 \sim 0.5$ solar, with a small scatter. Most other clusters, including merging clusters, also have similar Fe abundance profiles beyond $0.1 r_{180}$. These clusters may have universal metal enrichment histories, and a significant amount of Fe was synthesized at a very early stage in cluster formation. Mergers of clusters can destroy the central Fe peak.
\end{abstract}

Key words. galaxies: clusters: general - X-rays: galaxies: clusters - intergalactic medium

\section{Introduction}

Clusters of galaxies are the largest gravitationally bound objects in the universe. The intracluster medium (ICM) contains a large amount of metals, which are synthesized mainly by supernovae (SNe) in early-type galaxies (e.g., Arnaud et al. 1992; Renzini et al. 1993). Thus, metal abundances in the ICM provide important clues for understanding the metal-enrichment history and evolution of galaxies in clusters. Because both SN II and SN Ia synthesize Fe, the distribution of Fe in the ICM contains information about the star-formation histories of massive stars and the history of chemical-enrichment attributed to SN Ia.

The ASCA satellite (Tanaka et al. 1994) first enabled us to measure the distribution of Fe in the ICM (e.g., Fukazawa et al. 2000; Finoguenov et al. 2000, 2001). The Fe abundances of these clusters are $0.2-0.3$ solar, adopting the solar abundance from the "photospheric" values given by Anders \& Grevesse (1989). The dependence of the Fe abundance on the temperature of the ICM is weak (Fukazawa et al. 1998). ASCA revealed large-scale abundance gradients from AWM 7 and the Perseus cluster (Ezawa et al. 1997, 2001). De Grandi et al. (2004) derived the $\mathrm{Fe}$ abundances of nearby hot clusters observed with Beppo-SAX and found that the abundance profiles are strongly peaked for cool core clusters, whereas they remain constant for other systems. XMM-Newton and Chandra observations show a spatial distribution of the Fe abundance of up to $0.3 \sim 0.4 r_{180}$ (e.g., Tamura et al. 2004; Vikhlinin et al. 2005; Baldi et al. 2007; Maughan et al. 2008; Leccardi \& Molendi 2008). At the center of most relaxed clusters, the Fe abundance decreases steeply outward. Outside the central region, the Fe abundance decreases more gradually toward the outer regions.

The Suzaku satellite first measured the Fe abundance of the ICM beyond $0.5 r_{180}$. (Fujita et al. 2008; Tawa 2008). Within $0.3-0.5 r_{180}$, Suzaku can derive the Fe abundances more accurately than XMM. The Fe abundance gradually decreases from the center to $\sim 0.7 r_{180}$.

In this paper, we describe our study of the $\mathrm{Fe}$ abundance in the ICM up to $0.3 \sim 0.5 r_{180}$ of 28 brightest clusters of galaxies observed with XMM-Newton. Some clusters have cool cores at their center (e.g. Makishima et al. 2001; Böhringer et al. 2002). We must be careful when deriving elemental abundances with a multi-temperature plasma, because emission lines and a continuum spectrum are different functions of temperature. Therefore, we directly derived the strength of the $\mathrm{K} \alpha$ lines of $\mathrm{Fe}$, considering the temperature dependence of the flux ratios of the Fe lines and the continuum, and derived $\mathrm{Fe}$ abundances. We adopted an energy range in which the ICM dominates the background.

In Sect. 2 we summarize the observations and data preparation. Section 3 describes our analysis of the data, and in Sect. 4 the $\mathrm{Fe}$ abundances are determined. We discuss our results in Sect. 5.

We adopt the new solar abundances given by Lodders (2003), according to which the solar $\mathrm{Fe}$ abundance with regard to $H$ is $2.95 \times 10^{-5}$ by number. This value differs from the photospheric value $\left(4.68 \times 10^{-5}\right)$ given by Anders \& Grevesse (1989). Considering the difference in He abundance between the two solar abundance tables, the Fe abundance yielded by the former is 1.5 times higher than that of the latter. We use $H_{0}=$ $70 \mathrm{~km} \mathrm{~s}^{-1} \mathrm{Mpc}^{-1}$. Unless otherwise specified, errors are quoted with $68 \%$ confidence.

\section{Target selection and observations}

In the XMM-Newton archival data, we selected the 28 brightest clusters of galaxies with $z<0.08$ and total exposures above $7 \mathrm{ks}$ after filtering out background flares. The samples are listed in Table 1. The ICM temperatures in the "the X-ray Brightest Abell Clusters (XBACs)" by Ebeling et al. (1996) 
Table 1. Cluster samples in the XMM-Newton archival data.

\begin{tabular}{|c|c|c|c|c|c|}
\hline Cluster & \multicolumn{2}{|c|}{$\begin{array}{l}\langle T\rangle^{a} \\
(\mathrm{keV})\end{array}$} & \multicolumn{2}{|c|}{$\begin{array}{ll}N_{\mathrm{H}}^{b} & \text { Type }^{c} \\
\left(10^{20} \mathrm{~cm}^{-2}\right)\end{array}$} & \multirow{2}{*}{$\begin{array}{l}\begin{array}{l}\text { Obsid (exposures of PN, MOS1 and MOS2) } \\
(\mathrm{ks}, \mathrm{ks}, \mathrm{ks})\end{array} \\
0109980101(19.023 .623 .9) 0504780201(24.931 .633 .1)\end{array}$} \\
\hline A262 & 0.016 & 2.5 & 5.4 & $\mathrm{cD}$ & \\
\hline Virgo & 0.004 & 2.8 & 2.6 & $\mathrm{cD}$ & $\begin{array}{l}0114120101(30.037 .738 .5) 0200920101(69.676 .978 .5) \\
0106060101(5.09 .29 .2) 0106060201(1.65 .55 .0) 0106060301(5.47 .17 .9) \\
0141570101(17.423 .224 .7) 0112550701(1.00 .2) 0112552101(7.613 .413 .5) \\
0106060401(7.310 .711 .1) 0145800101(5.937 .80 .20 .2) 0106060501(10.915 .315 .4) \\
0200650101(42.753 .253 .5) 0112840101(14.618 .117 .6) 0106060601(8.610 .510 .7) \\
0112550801(2.913 .212 .1) 0106060701(1.212 .45 .9) 0108860101(16.720 .619 .2) \\
0106061401(4.98 .08 .3)\end{array}$ \\
\hline A4038 & 0.029 & 3.0 & 1.5 & $\mathrm{cD}$ & $0204460101(27.829 .328 .9)$ \\
\hline A1060 & 0.012 & 3.0 & 4.9 & non-cD & $0206230101(28.537 .938 .9)$ \\
\hline A2052 & 0.035 & 3.1 & 3.0 & $\mathrm{cD}$ & $0109920101(26.428 .929 .0)$ \\
\hline A1367 & 0.021 & 3.3 & 2.4 & non-cD & $0061740101(23.731 .831 .4) 0005210101$ (20.8 27.828 .8$)$ \\
\hline A780 & 0.057 & 3.5 & 4.7 & $\mathrm{cD}$ & $0109980301(14.019 .122 .2)$ \\
\hline A2589 & 0.042 & 3.6 & 3.9 & $\mathrm{cD}$ & 0204180101 (21.8 26.327 .5$)$ \\
\hline AWM7 & 0.017 & 3.6 & 9.8 & $\mathrm{cD}$ & $0135950301(27.530 .730 .9)$ \\
\hline MKW3s & 0.045 & 3.7 & 3.0 & $\mathrm{cD}$ & $0109930101(0.037 .738 .0)$ \\
\hline A2063 & 0.036 & 3.9 & 3.0 & $\mathrm{cD}$ & $0200120401(5.08 .25 .0)$ \\
\hline A3526 & 0.010 & 4.0 & 8.2 & $\mathrm{cD}$ & $\begin{array}{l}0046340101(35.244 .943 .5) 0406200101(90.5111 .7111 .7) 0504360101 \text { (26.4 } 28.2 \text { 30.9) } \\
0504360201(32.934 .034 .6)\end{array}$ \\
\hline A2199 & 0.030 & 4.2 & 0.9 & $\mathrm{cD}$ & $0008030601(0.56 .94 .7) 0008030301(2.94 .74 .7) 0008030201(13.314 .514 .6)$ \\
\hline A496 & 0.033 & 4.4 & 4.6 & $\mathrm{cD}$ & 0135120201 (11.8 16.215 .9$) 0506260301$ (44.5 57.0 59.5) 0506260401 (44.8 55.858 .6$)$ \\
\hline A1644 & 0.048 & 4.6 & 4.8 & non-cD & $0010420201(12.714 .114 .1)$ \\
\hline A3562 & 0.050 & 4.8 & 4.0 & non-cD & 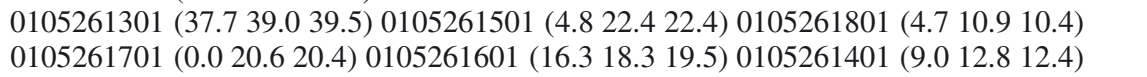 \\
\hline A3558 & 0.048 & 5.4 & 4.2 & non-cD & $0107260101(38.243 .142 .5)$ \\
\hline A3627 & 0.016 & 5.5 & 20.8 & non-cD & 0204250101 (2.4 6.2 5.8) 0208010101 (9.1 12.6 13.0) 0208010201 (10.9 13.614 .3$)$ \\
\hline A1795 & 0.062 & 5.8 & 1.2 & $\mathrm{cD}$ & $\begin{array}{l}0097820101(25.138 .337 .9) 0109070201(53.054 .554 .6) 0205190101(26.929 .528 .8) \\
0205190201(20.822 .623 .2)\end{array}$ \\
\hline A85 & 0.052 & 5.8 & 3.1 & $\mathrm{cD}$ & $0065140101(9.812 .412 .2) 0065140201(9.212 .112 .4)$ \\
\hline A3667 & 0.053 & 6.0 & 4.6 & non-cD & $\begin{array}{l}0105260101(0.87 .36 .8) 0206850101(52.359 .260 .0) 0105260601(17.723 .123 .3) \\
0105260301(12.817 .016 .2) 0105260401(11.516 .316 .5) 0105260501(11.212 .612 .6) \\
0105260201(15.119 .018 .4)\end{array}$ \\
\hline $\begin{array}{l}\text { A } 426 \\
\text { (Perseus) }\end{array}$ & 0.018 & 6.1 & 14.6 & $\mathrm{cD}$ & $\begin{array}{l}0085110101(47.548 .850 .7) 0204720101(11.915 .115 .2) 0085590201(38.441 .740 .0) \\
0305720101(10.013 .012 .6) 0305720301(15.919 .921 .2) 0151560101(18.024 .925 .1) \\
0204720201 \text { (20.7 24.5 24.5) } 0405410201(9.417 .325 .5) 0405410101(12.218 .216 .1)\end{array}$ \\
\hline A2256 & 0.058 & 6.3 & 4.1 & non-cD & $\begin{array}{l}0141380101(6.49 .28 .7) 0141380201(10.613 .212 .4) \\
0112951501(5.910 .610 .9) 0112951601(7.212 .712 .7) 0112950601(8.412 .311 .8) \\
0112500101(21.825 .0 \text { 25.2) }\end{array}$ \\
\hline A3571 & 0.040 & 6.5 & 4.4 & $\mathrm{cD}$ & 0086950201 (14.3 25.425 .2$)$ \\
\hline A2029 & 0.077 & 7.4 & 3.2 & $\mathrm{cD}$ & $0111270201(9.312 .112 .4)$ \\
\hline $\begin{array}{l}\text { A1656 } \\
\text { (Coma) }\end{array}$ & 0.023 & 7.8 & 0.9 & non-cD & $\begin{array}{l}0300530201(0.43 .63 .4) 0153750101(19.221 .321 .8) 0124711401(14.717 .416 .5) \\
0300530101(18.620 .921 .0) 0300530301(26.530 .530 .2) 0300530701(16.424 .424 .6) \\
0300530401(13.318 .819 .6) 0300530601(16.722 .622 .1) 0300530501(19.224 .724 .6) \\
0124710901(16.721 .622 .1) 0124712001(9.215 .414 .6) 0124710501(10.724 .424 .8) \\
0124710601(4.79 .59 .0) 0204040101(69.773 .074 .0) 0204040301(43.752 .953 .2) \\
0204040201(54.861 .864 .8) 0124710801(18.424 .524 .5) 0124710401(8.25 .95 .6) \\
0124712501(23.827 .727 .7) 0124712401(11.017 .418 .1) 0124711101(13.219 .321 .1) \\
0124712201(18.226 .726 .2) 0124712101(20.326 .026 .1) 0124710301(10.613 .414 .0) \\
0124710101(23.730 .630 .8)\end{array}$ \\
\hline A3266 & 0.055 & 8.4 & 1.5 & non-cD & $\begin{array}{l}0105262201(3.40 .03 .6) 0105260901(18.624 .424 .3) 0105260801(16.219 .620 .4) \\
0105262501(3.57 .67 .7) 0105262001(2.97 .67 .6) 0105260701(16.120 .620 .5) \\
0105261101(8.012 .711 .1) 0105262101(4.45 .67 .2)\end{array}$ \\
\hline A754 & 0.054 & 8.6 & 4.7 & non-cD & $\begin{array}{l}0112950301(8.013 .313 .7) 0112950401(7.614 .314 .3) 0136740201(2.36 .06 .7) \\
0136740101(12.514 .214 .2)\end{array}$ \\
\hline
\end{tabular}

Notes. ${ }^{(a)}$ Adopted average temperature of ICM used to calculate $r_{180} .{ }^{(b)}$ The Galactic value of the hydrogen column density (Dickey \& Lockman 1990). (c) "cD" corresponds to cD clusters, which are relaxed clusters with a cD galaxy at their center. Other clusters are classified as "non-cD." (d) Obsid (a unique XMM-Newton observation identifier) and exposure times of PN, MOS1, and MOS2, respectively, after screening out the background flares.

range from $2.4 \mathrm{keV}$ to $8.7 \mathrm{keV}$. Seventeen clusters show relatively relaxed morphologies with a $\mathrm{cD}$ galaxy at the X-ray peak. Hereafter, these are called cD clusters. Eleven clusters are merging clusters or relaxed clusters with two giant galaxies in the central region such as the Coma cluster. Hereafter, these clusters are called non-cD clusters. 
The virial radius of each cluster, $r_{180}=$ $1.95 h_{100}^{-1} \sqrt{k\langle T\rangle / 10 \mathrm{keV}}$, was calculated following Markevitch et al. (1998), by adopting an average temperature $\langle T\rangle$ of that at $0.06-0.3 r_{180}$ from the X-ray peak of each cluster.

We used the PN, MOS1, and MOS2 detectors to derive the Fe abundance of the ICM. We selected events with patterns smaller than 5 and 13 for the PN and MOS, respectively.

Spectra were accumulated in concentric annular regions of $0-0.03 r_{180}, 0.03-0.06 r_{180}, 0.06-0.1 r_{180}, 0.1-0.2 r_{180}$, $0.2-0.3 r_{180}$, and $0.3-0.5 r_{180}$ centered on the X-ray peak of each cluster. Here, the X-ray peaks were derived using the ewavelet tool of SAS-v8.0.0, and luminous point sources were excluded. The calculated X-ray peak of each cluster and the accumulation regions for the spectra are summarized as X-ray images in Fig. 1. The spectra from MOS1 and MOS2 were added. The background spectrum was calculated by integrating blank-sky data in the same detector regions. From deep-sky observations with the XMM, we selected the data with the background most similar to that of each cluster, and the faintest Galactic emission, after screening out background flare events in the data and the background, following Katayama et al. (2004). Then, the background was scaled using the count rate between 10 and $12 \mathrm{keV}$. Most scaling factors are smaller than $10 \%$, because we selected a blank-sky observation to match each cluster observation.

The response matrix file and the auxiliary response file (ARF) corresponding to each spectrum were calculated using SAS-v8.0.0. We used the XSPEC_v11.3.2ag package for our spectral analysis.

\section{Analysis}

\subsection{Fe line strength}

In this study, the Fe abundances of the ICM are derived from the ratio of the flux in units of photons $\mathrm{cm}^{-2} \mathrm{~s}^{-1}$ of the $\mathrm{K} \alpha$ lines of He-like Fe (hereafter $F_{\mathrm{FeHeK}}$ ) or $\mathrm{H}$-like $\mathrm{Fe}$ (hereafter $F_{\mathrm{FeHK}}$ ) to that of the continuum at $3.5-6.0 \mathrm{keV}$ (hereafter $F_{3.5-6}$ ), since the systematic uncertainty in the continuum flux in this energy band owing to the background is smaller than that around the Fe-K lines. Another advantage is that the dependence of the ratios on the plasma temperature is relatively weak within a certain temperature range. The temperature dependence of these ratios can constrain on the $\mathrm{Fe}$ abundances of multi-temperature plasmas.

To obtain suitable statistics, we simultaneously fitted the raw annular spectra of a cluster and a deep field within an energy range of 5.0-7.2 keV with the sum of the ICM and background emission. The ICM component consists of thermal bremsstrahlung and two Gaussians for the $\mathrm{K} \alpha$ lines of $\mathrm{He}-$ like and $\mathrm{H}$-like Fe. We modeled the background emission with a power-law model for the cosmic X-ray background (CXB), a "powerlaw/b" model for non X-ray background (NXB), and three Gaussians at $5.4 \mathrm{keV}, 5.9 \mathrm{keV}$, and $6.4 \mathrm{keV}$, which are the $\mathrm{K} \alpha$ lines of neutral $\mathrm{Cr}, \mathrm{Mn}$, and Fe, respectively. The "powerlaw/b" model is not folded through the ARF, and differs from a power-law. We obtained acceptable fits with a reduced $\chi^{2} \sim$ 1. Figure 2 shows representative spectra of Abell 426 (A426, the Perseus cluster) fitted in this way. These spectra are fitted well, and the $\mathrm{K} \alpha$ line of He-like Fe is clearly detected even at $0.3-0.5 r_{180}$. The background line at $6.4 \mathrm{keV}$ may cause a systematic uncertainty in the strength of the He-like Fe line in the MOS spectra at low-brightness regions. In contrast, the background lines in the PN spectra in this energy range are much weaker than those in the MOS.
Despite the $6.4 \mathrm{keV}$ background line, the PN and MOS gave mostly consistent ratios of $F_{\mathrm{FeHeK}} / F_{3.5-6}$ and $F_{\mathrm{FeHK}} / F_{3.5-6}$. Hereafter we used the weighted average of these ratios from the PN and MOS to derive Fe abundances with relative errors smaller than $30 \%$.

\subsection{Spectral fit with single-temperature model}

To convert the observed line flux to the Fe abundance, we derived the ICM temperatures. The first step in this analysis was to fit the background-subtracted annular (projected) spectra from the PN and MOS with a single-temperature vAPEC model (Smith et al. 2001) with photoelectric absorption fixed at the Galactic value (hereafter the 1T model). The energy ranges of 1.4-1.6 keV for the MOS and 7.2-9.0 keV for the PN were discarded owing to strong instrumental lines. To account for the remaining background, we added a "powerlaw/b" model (Zhang et al. 2009) and fitted the spectra within the energy range of $1.2-10.0 \mathrm{keV}$. Here, we fixed the photon index of the "powerlaw/b" component at 0.15 and 0.35 for the MOS and PN, respectively (Zhang et al. 2009). The abundances of $\mathrm{C}, \mathrm{N}$, and $\mathrm{Al}$ were fixed at 1 solar and those of $\mathrm{O}$ and $\mathrm{Ne}$ were assumed to have the same values as $\mathrm{Mg}$. The abundances of $\mathrm{Mg}, \mathrm{Si}, \mathrm{S}, \mathrm{Ar}, \mathrm{Ca}, \mathrm{Fe}$, and Ni were allowed to vary. We did not use a more detailed modeling of the background (Snowden et al. 2008; Leccardi \& Molendi 2008), since we mainly used an energy range over which the ICM emission is dominant. The ICM temperatures derived from the PN and MOS are consistent with each other within the order of several percent. We adopted the weighted average of the temperatures derived from the PN and MOS, as summarized in Table 2.

To derive the Fe abundance in each annular region, we included a $10 \%$ systematic uncertainty in temperatures, considering the uncertainties described below. When the spectra are fitted within the energy range of $0.8-10.0 \mathrm{keV}$, which includes Fe$\mathrm{L}$ emission, the derived temperatures decreased systematically by $\sim 10 \%$. We also fitted the spectra without the "powerlaw/b" model. Then, outside $0.2 r_{180}$, the derived ICM temperature increased by $10 \sim 20 \%$ and the discrepancy between the PN and MOS became increased. Similarly, we also analyzed the same XMM data in the same way with SAS v7.0.0. In this case, when the derived temperatures are higher than several $\mathrm{keV}$, the MOS systematically gives $\sim 10 \%$ higher than those given by the PN. Systematic differences of $\sim 10 \%$ in cluster temperature among Chandra, the PN, and the MOS were reported in relatively hightemperature clusters (Snowden et al. 2008). Reese et al. (2010) found that the ICM temperature derived from Chandra data changes systematically by $\sim 10 \%$ between calibrations.

\subsection{Spectral fit with multi-temperature model}

To study the effect of the temperature structure of the ICM, we also fitted the background-subtracted spectra of the MOS at $0.5-10.0 \mathrm{keV}$, using a multi-temperature vAPEC model with photoelectric absorption (multi-T model), the Galactic emission, and the "powerlaw/b" model with a fixed photon index, as described in Sect. 3.2. The Galactic emission, which includes the local hot bubble, the Milky Way halo, and solar wind charge exchange, is empirically fitted with a two-temperature APEC model with redshift $=0$ (e.g. Lumb et al. 2002; Yoshino et al. 2009). Therefore, we used the two-temperature APEC model for the Galactic emission. The temperature of the one component was fixed at $0.1 \mathrm{keV}$, and that of the other was restricted within 0.2-0.4 keV. Here, we used only the MOS detector, because 

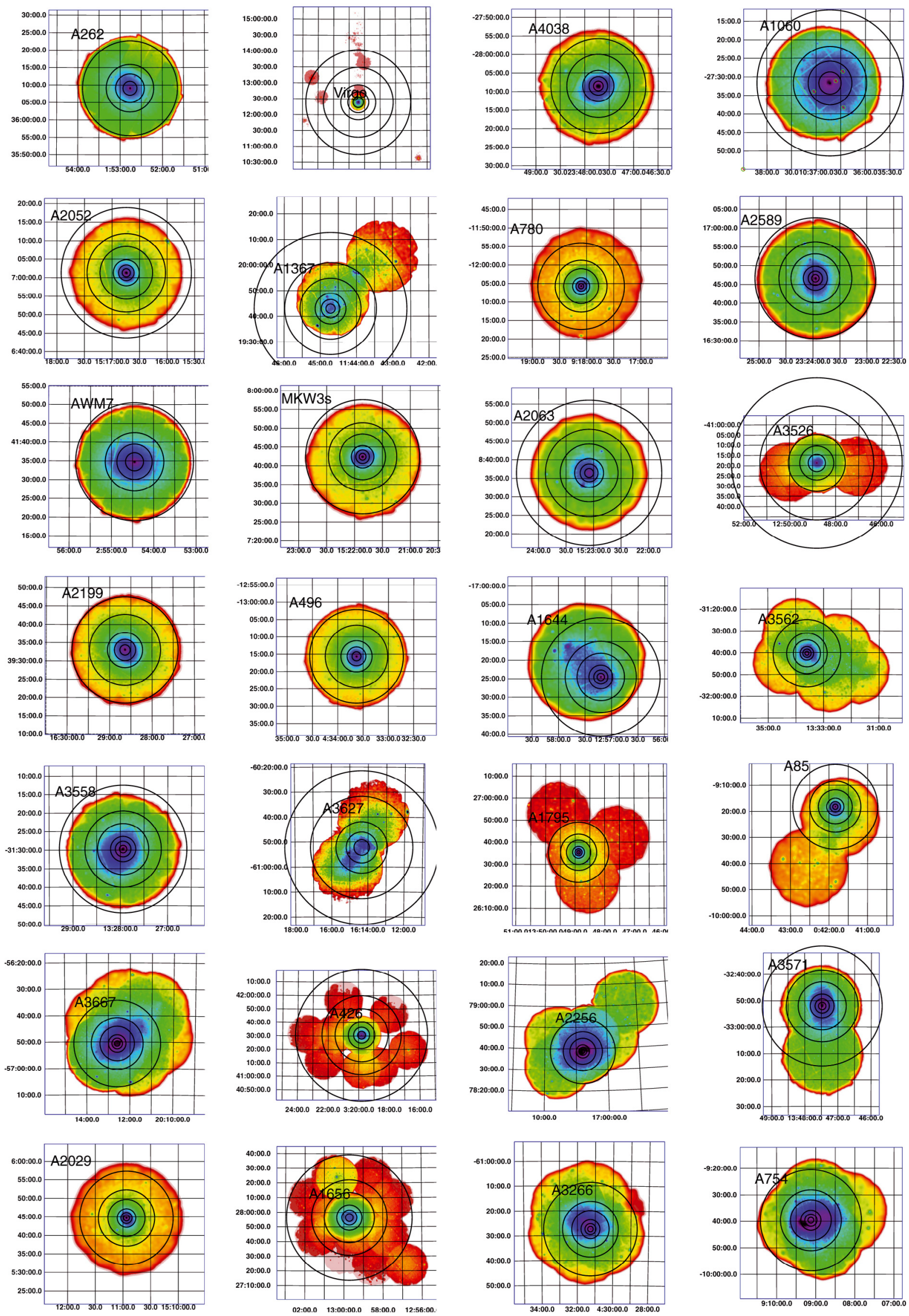

Fig. 1. Combined MOS and PN images of the target clusters. Exposures are not corrected and therefore the exposures of each field can be visualized. Circles have radii of $0.03,0.06,0.1,0.2,0.3$, and $0.5 r_{180}$. 

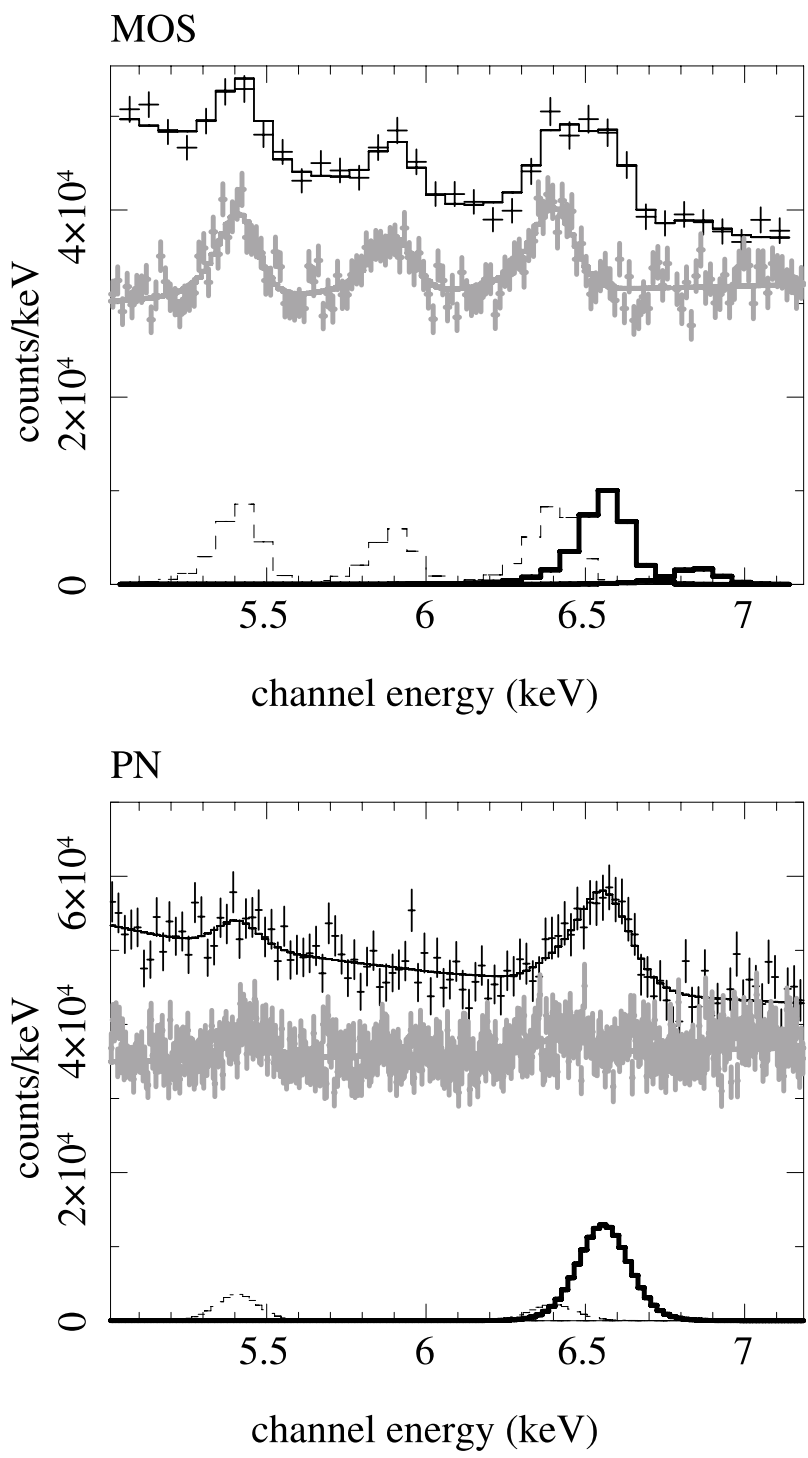

Fig. 2. Representative spectra of Abell 426 (the Perseus cluster) (black) and the adopted background (gray) at $0.3-0.5 r_{180}$ from MOS and PN fitted with Gaussians and continuum. Best-fit Gaussians corresponding to the $\mathrm{K} \alpha$-lines of He-like and $\mathrm{H}$-like Fe are plotted as bold lines: those for background lines are plotted as thin dashed lines.

it has a better energy resolution and is more sensitive to temperature structure than the PN detector. The multi-T model is a sum of 13 temperature components, because the spectrum of an isothermal plasma can be reproduced well by the sum of the spectra of two neighboring temperature plasmas with similar elemental abundances. The ratio of the temperatures of the two neighboring components is fixed at 0.8 . The hottest temperature of the multi-T model is fixed at the highest temperature derived using the $1 \mathrm{~T}$ model derived from the MOS within $0.2 r_{180}$. The elemental abundances of each element for the multi-T components are assumed to have the same values. We added a $3 \%$ systematic error to the count rate of each channel in the spectra. Then, the reduced $\chi^{2}$ reaches the reasonable values of $\sim 1$.

\section{Results}

\subsection{Temperature profiles of the ICM}

The radial temperature profiles of the ICM are summarized in Fig. 3. From $0.06 r_{180}$ to $0.2 \sim 0.3 r_{180}$, the temperature gradient of the ICM is flat, whereas the temperatures of several cD clusters are lower within $0.06 r_{180}$ than those at $0.06-0.2 r_{180}$, owing to the presence of cool cores. In contrast, most non-cD clusters have flatter radial temperature profiles within $0.2 \sim 0.3 r_{180}$. Beyond $0.2 \sim 0.3 r_{180}$, the ICM temperatures tend to decrease outward. These temperature profiles are consistent with previous Chandra and XMM-Newton measurements (e.g., Snowden et al. 2008; Vikhlinin et al. 2005).

\subsection{Fe abundance of the ICM}

Figure 4 shows the observed values of $F_{\mathrm{FeHeK}} / F_{3.5-6}$ and $F_{\mathrm{FeHK}} / F_{3.5-6}$, plotted against the ICM temperature derived from the $1 \mathrm{~T}$ model. Within $0.03 r_{180}, F_{\mathrm{FeHeK}} / F_{3.5-6}$ is scattered from 0.03 to 0.2 , with mild temperature dependence. Beyond $0.03 r_{180}$, both $F_{\mathrm{FeHeK}} / F_{3.5-6}$ and $F_{\mathrm{FeHK}} / F_{3.5-6}$ become closer to the theoretical relationships predicted by the APEC model assuming that the $\mathrm{Fe}$ abundance $=0.5$ solar. Beyond $0.3 r_{180}$, the data for only several clusters have adequate statistics: the $F_{\mathrm{FeHeK}} / F_{3.5-6}$ values of A426 (the Perseus cluster) and a non-cD cluster are close to the relationship for 0.5 solar, whereas those of two non-cD clusters are significantly lower.

The temperature dependence of the theoretical ratio $F_{\mathrm{FeHeK}} / F_{3.5-6}$ for a given $\mathrm{Fe}$ abundance is fairly weak, within $20 \%$ for $2-6 \mathrm{keV}$, and starts to decrease above $6 \mathrm{keV}$ (Fig. 4). In contrast, the ratio of $F_{\mathrm{FeHK}} / F_{3.5-6}$ is nearly constant within $20 \%$ for $6.7-17 \mathrm{keV}$. This temperature independence minimizes the systematic uncertainty in the Fe abundance because of uncertainties in the temperature structure.

To derive the Fe abundance, we converted the observed ratio of $F_{\mathrm{FeHeK}} / F_{3.5-6}$ using the theoretical ratios calculated by the APEC model and the ICM temperature derived from the 1T model. The results are summarized in Table 2.

\subsection{Radial profiles of $\mathrm{Fe}$ abundances}

The radial profiles of the Fe abundances in the ICM are shown in Fig. 5. Within $0.03 r_{180}$, the derived Fe abundances of the cD clusters are $0.7-1.6$ solar. Non-cD clusters with large deviations from spherical symmetry, A1367 and A3562, also have high Fe abundances of $\sim 1.5$ solar. In contrast, the other non-cD clusters have lower central values of Fe. Outside $0.1 r_{180}$, both the $\mathrm{cD}$ and non-cD clusters have similar radial profiles of the $\mathrm{Fe}$ abundance. At $0.1-0.2 r_{180}$, the derived Fe abundances are $\sim 0.5$ solar. For the region at $0.3-0.5 r_{180}$, although sufficient statistics are available for only six clusters, the derived $\mathrm{Fe}$ abundances are around $0.3-0.5$ solar.

Figure 6 shows the ratios of the Fe abundances of each cluster at each annular region to that at $0.06-0.1 r_{180}$. Except for the Centaurus cluster (A3526), the Fe abundances of the cD clusters have similar slopes: the $\mathrm{Fe}$ abundances within $0.03 r_{180}$ are higher than those at $0.06-0.1 r_{180}$ by a factor of 1.5 . Beyond $0.06 r_{180}$, the radial profiles become flatter.

The Fe abundances of three morphologically distorted clusters, A1367, A3558, and A3562, within $0.03 r_{180}$ are higher than those at $0.06-0.1 r_{180}$ by a factor of $1.5-2$. The other non-cD clusters have similar Fe abundances within $0.03 r_{180}$ and $0.06-0.1 r_{180}$.

\subsection{Temperature dependence of the Fe abundance}

In Fig. 7 the derived Fe abundances are plotted against the ICM temperature. There is no significant dependence on the plasma 
Table 2. Results of spectral fitting of annular spectra.

\begin{tabular}{|c|c|c|c|c|}
\hline Cluster & $r\left(r_{180}\right)$ & $k T^{a}(\mathrm{keV})$ & $\begin{array}{l}\mathrm{Fe} \text { (solar) from } F_{\mathrm{FeHeK}} / F_{3.5-6} \\
\text { Best-fit }{ }^{b} \pm \Delta_{\text {stat }^{c}}{ }^{c} \Delta_{\text {sys }}{ }^{d}\end{array}$ & $\begin{array}{l}\mathrm{Fe} \text { (solar) from } F_{\mathrm{FeHK}} / F_{3.5-6} \\
\text { Best-fit }^{b} \pm \Delta_{\text {stat }}{ }^{c} \Delta_{\text {sys }}\end{array}$ \\
\hline A262 & $0.00-0.03$ & $1.95 \pm 0.03$ & $1.31 \pm 0.28-0.26$ & - \\
\hline A262 & $0.03-0.06$ & $2.36 \pm 0.04$ & $0.76 \pm 0.09-0.03$ & - \\
\hline A262 & $0.06-0.10$ & $2.43 \pm 0.06$ & $0.62 \pm 0.08-0.02$ & - \\
\hline A262 & $0.10-0.20$ & $2.12 \pm 0.08$ & $0.90 \pm 0.21-0.13$ & - \\
\hline Virgo & $0.00-0.03$ & $2.11 \pm 0.00$ & $0.86 \pm 0.09-0.11$ & - \\
\hline Virgo & $0.03-0.06$ & $2.47 \pm 0.01$ & $0.52 \pm 0.05-0.02$ & - \\
\hline A4038 & $0.00-0.03$ & $3.12 \pm 0.06$ & $0.79 \pm 0.06+0.00$ & - \\
\hline A4038 & $0.03-0.06$ & $3.22 \pm 0.08$ & $0.66 \pm 0.05+0.00$ & - \\
\hline A4038 & $0.06-0.10$ & $3.04 \pm 0.07$ & $0.57 \pm 0.05+0.00$ & - \\
\hline A4038 & $0.10-0.20$ & $2.81 \pm 0.10$ & $0.55 \pm 0.08+0.01$ & - \\
\hline A1060 & $0.00-0.03$ & $3.35 \pm 0.03$ & $0.67 \pm 0.03-0.01$ & - \\
\hline A1060 & $0.03-0.06$ & $3.27 \pm 0.04$ & $0.55 \pm 0.02-0.00$ & - \\
\hline A1060 & $0.06-0.10$ & $2.93 \pm 0.05$ & $0.52 \pm 0.05-0.01$ & - \\
\hline A1060 & $0.10-0.15$ & $2.79 \pm 0.12$ & $0.46 \pm 0.10-0.01$ & - \\
\hline A2052 & $0.00-0.03$ & $2.57 \pm 0.04$ & $1.28 \pm 0.17-0.07$ & - \\
\hline A2052 & $0.03-0.06$ & $3.12 \pm 0.06$ & $0.84 \pm 0.05-0.00$ & - \\
\hline A2052 & $0.06-0.10$ & $3.12 \pm 0.07$ & $0.81 \pm 0.06-0.00$ & - \\
\hline A2052 & $0.10-0.20$ & $3.01 \pm 0.07$ & $0.69 \pm 0.06-0.01$ & - \\
\hline A1367 & $0.00-0.03$ & $2.83 \pm 0.23$ & $1.71 \pm 0.45-0.15$ & - \\
\hline A1367 & $0.03-0.06$ & $3.37 \pm 0.18$ & $0.78 \pm 0.11+0.00$ & - \\
\hline A1367 & $0.06-0.10$ & $3.33 \pm 0.17$ & $0.71 \pm 0.09-0.01$ & - \\
\hline A1367 & $0.10-0.20$ & $3.36 \pm 0.14$ & $0.45 \pm 0.07+0.00$ & - \\
\hline A1367 & $0.20-0.30$ & $3.22 \pm 0.30$ & $0.71 \pm 0.17-0.02$ & - \\
\hline A780 & $0.00-0.03$ & $3.41 \pm 0.09$ & $0.72 \pm 0.06+0.02$ & - \\
\hline A780 & $0.03-0.06$ & $3.67 \pm 0.10$ & $0.56 \pm 0.05+0.01$ & - \\
\hline A780 & $0.06-0.10$ & $3.62 \pm 0.12$ & $0.41 \pm 0.07+0.01$ & - \\
\hline A780 & $0.10-0.20$ & $3.93 \pm 0.12$ & $0.36 \pm 0.04+0.00$ & - \\
\hline A780 & $0.20-0.30$ & $4.33 \pm 0.39$ & $0.41 \pm 0.13-0.02$ & - \\
\hline A2589 & $0.00-0.03$ & $3.48 \pm 0.15$ & $1.50 \pm 0.17-0.01$ & - \\
\hline A2589 & $0.03-0.06$ & $3.59 \pm 0.14$ & $1.06 \pm 0.10-0.01$ & - \\
\hline A2589 & $0.06-0.10$ & $3.50 \pm 0.14$ & $0.92 \pm 0.10-0.01$ & - \\
\hline A2589 & $0.10-0.20$ & $3.34 \pm 0.14$ & $0.79 \pm 0.10-0.01$ & - \\
\hline AWM7 & $0.00-0.03$ & $3.44 \pm 0.05$ & $1.12 \pm 0.06+0.01$ & - \\
\hline AWM7 & $0.03-0.06$ & $3.78 \pm 0.07$ & $0.89 \pm 0.04+0.00$ & - \\
\hline AWM7 & $0.06-0.10$ & $3.70 \pm 0.08$ & $0.62 \pm 0.03+0.00$ & - \\
\hline AWM7 & $0.10-0.19$ & $3.67 \pm 0.10$ & $0.63 \pm 0.04+0.00$ & - \\
\hline MKW3s & $0.00-0.03$ & $3.18 \pm 0.10$ & $1.06 \pm 0.11+0.01$ & - \\
\hline MKW3s & $0.03-0.06$ & $3.67 \pm 0.12$ & $0.88 \pm 0.08+0.00$ & - \\
\hline MKW3s & $0.06-0.10$ & $3.78 \pm 0.15$ & $0.57 \pm 0.06+0.00$ & - \\
\hline MKW3s & $0.10-0.20$ & $3.47 \pm 0.15$ & $0.55 \pm 0.08+0.01$ & - \\
\hline A2063 & $0.00-0.03$ & $3.29 \pm 0.20$ & $1.02 \pm 0.32-0.01$ & - \\
\hline A2063 & $0.03-0.06$ & $3.66 \pm 0.21$ & $1.04 \pm 0.20+0.01$ & - \\
\hline A2063 & $0.06-0.10$ & $4.05 \pm 0.39$ & $0.61 \pm 0.14-0.01$ & - \\
\hline A3526 & $0.00-0.03$ & $2.58 \pm 0.01$ & $1.63 \pm 0.18-0.14$ & - \\
\hline A3526 & $0.03-0.06$ & $3.90 \pm 0.03$ & $0.72 \pm 0.02+0.00$ & - \\
\hline A 3526 & $0.06-0.10$ & $3.81 \pm 0.03$ & $0.57 \pm 0.03+0.01$ & - \\
\hline A3526 & $0.10-0.20$ & $3.19 \pm 0.12$ & $0.36 \pm 0.03+0.01$ & - \\
\hline A2199 & $0.00-0.03$ & $3.63 \pm 0.05$ & $0.78 \pm 0.04+0.04$ & - \\
\hline A2199 & $0.03-0.06$ & $4.25 \pm 0.08$ & $0.60 \pm 0.04-0.01$ & - \\
\hline A2199 & $0.06-0.10$ & $4.28 \pm 0.12$ & $0.60 \pm 0.04-0.01$ & - \\
\hline A2199 & $0.10-0.20$ & $4.33 \pm 0.11$ & $0.45 \pm 0.04-0.01$ & - \\
\hline A2199 & $0.20-0.30$ & $4.18 \pm 0.46$ & $0.50 \pm 0.13-0.01$ & - \\
\hline A496 & $0.00-0.03$ & $3.01 \pm 0.02$ & $0.93 \pm 0.05+0.08$ & - \\
\hline A496 & $0.03-0.06$ & $4.16 \pm 0.03$ & $0.70 \pm 0.03+0.05$ & - \\
\hline A496 & $0.06-0.10$ & $4.72 \pm 0.05$ & $0.61 \pm 0.04+0.01$ & - \\
\hline A496 & $0.10-0.20$ & $4.73 \pm 0.08$ & $0.48 \pm 0.04+0.01$ & - \\
\hline A496 & $0.20-0.30$ & $3.94 \pm 0.17$ & $0.34 \pm 0.05+0.03$ & - \\
\hline A1644 & $0.03-0.06$ & $4.47 \pm 0.43$ & $0.76 \pm 0.22+0.04$ & - \\
\hline A1644 & $0.06-0.10$ & $4.89 \pm 0.35$ & $0.84 \pm 0.17+0.01$ & - \\
\hline A1644 & $0.10-0.20$ & $4.95 \pm 0.30$ & $0.64 \pm 0.11-0.00$ & - \\
\hline A1644 & $0.20-0.30$ & $4.20 \pm 0.31$ & $0.61 \pm 0.15+0.04$ & - \\
\hline A 3562 & $0.00-0.03$ & $4.62 \pm 0.24$ & $1.37 \pm 0.17-0.01$ & - \\
\hline A 3562 & $0.03-0.06$ & $4.71 \pm 0.20$ & $0.90 \pm 0.09+0.01$ & - \\
\hline A3562 & $0.06-0.10$ & $4.85 \pm 0.20$ & $0.75 \pm 0.09+0.02$ & - \\
\hline A3562 & $0.10-0.20$ & $4.63 \pm 0.18$ & $0.63 \pm 0.06+0.02$ & - \\
\hline
\end{tabular}


Table 2. continued.

\begin{tabular}{|c|c|c|c|c|}
\hline Cluster & $r\left(r_{180}\right)$ & $k T^{a}(\mathrm{keV})$ & $\begin{array}{l}\mathrm{Fe} \text { (solar) from } F_{\mathrm{FeHeK}} / F_{3.5-6} \\
\text { Best-fit }^{b} \pm \Delta_{\text {stat }}{ }^{c} \Delta_{\text {sys }}{ }^{d}\end{array}$ & $\begin{array}{l}\text { Fe (solar) from } F_{\mathrm{FeHK}} / F_{3.5-6} \\
\text { Best-fit }^{b} \pm \Delta_{\text {stat }^{c}}{ }^{d} \Delta_{\text {sys }}\end{array}$ \\
\hline A3562 & $0.20-0.30$ & $4.49 \pm 0.34$ & $0.52 \pm 0.14+0.02$ & - \\
\hline A3558 & $0.00-0.03$ & $5.07 \pm 0.17$ & $0.75 \pm 0.07+0.07$ & - \\
\hline A3558 & $0.03-0.06$ & $5.88 \pm 0.17$ & $0.64 \pm 0.07-0.01$ & $0.83 \pm 0.19-0.01$ \\
\hline A3558 & $0.06-0.10$ & $5.87 \pm 0.16$ & $0.50 \pm 0.06-0.01$ & $0.49 \pm 0.13-0.01$ \\
\hline A3558 & $0.10-0.20$ & $5.29 \pm 0.11$ & $0.46 \pm 0.04+0.02$ & $0.53 \pm 0.14-0.07$ \\
\hline A3558 & $0.20-0.30$ & $4.77 \pm 0.16$ & $0.45 \pm 0.05+0.07$ & - \\
\hline A3627 & $0.00-0.03$ & $5.76 \pm 0.39$ & $0.51 \pm 0.09-0.00$ & - \\
\hline A3627 & $0.03-0.06$ & $5.74 \pm 0.29$ & $0.49 \pm 0.07+0.01$ & - \\
\hline A3627 & $0.06-0.10$ & $6.11 \pm 0.20$ & $0.44 \pm 0.06-0.00$ & - \\
\hline A3627 & $0.10-0.20$ & $4.97 \pm 0.17$ & $0.52 \pm 0.06+0.06$ & - \\
\hline A3627 & $0.20-0.30$ & $4.04 \pm 0.44$ & $0.73 \pm 0.17+0.17$ & - \\
\hline A1795 & $0.00-0.03$ & $4.21 \pm 0.05$ & $0.86 \pm 0.04+0.11$ & - \\
\hline A1795 & $0.03-0.06$ & $5.32 \pm 0.10$ & $0.73 \pm 0.06+0.02$ & $0.94 \pm 0.23-0.04$ \\
\hline A1795 & $0.06-0.10$ & $5.95 \pm 0.14$ & $0.56 \pm 0.07+0.02$ & $0.54 \pm 0.14-0.06$ \\
\hline A1795 & $0.10-0.20$ & $6.21 \pm 0.16$ & $0.43 \pm 0.05-0.01$ & $0.34 \pm 0.09+0.00$ \\
\hline A1795 & $0.20-0.30$ & $6.20 \pm 0.27$ & $0.57 \pm 0.09+0.00$ & - \\
\hline A1795 & $0.30-0.50$ & $5.72 \pm 0.62$ & $0.35 \pm 0.10+0.03$ & - \\
\hline A 85 & $0.00-0.03$ & $4.24 \pm 0.15$ & $0.98 \pm 0.09+0.09$ & - \\
\hline A85 & $0.03-0.06$ & $5.69 \pm 0.23$ & $0.84 \pm 0.11-0.01$ & - \\
\hline A85 & $0.06-0.10$ & $5.92 \pm 0.36$ & $0.61 \pm 0.10-0.04$ & - \\
\hline A85 & $0.10-0.20$ & $6.11 \pm 0.32$ & $0.52 \pm 0.08-0.02$ & - \\
\hline A85 & $0.20-0.30$ & $5.27 \pm 0.52$ & $0.49 \pm 0.12+0.05$ & - \\
\hline A3667 & $0.00-0.03$ & $6.61 \pm 0.30$ & $0.61 \pm 0.10-0.00$ & $0.85 \pm 0.19-0.05$ \\
\hline A3667 & $0.03-0.06$ & $6.41 \pm 0.16$ & $0.49 \pm 0.07+0.01$ & $0.59 \pm 0.14-0.04$ \\
\hline A3667 & $0.06-0.10$ & $6.33 \pm 0.14$ & $0.51 \pm 0.06+0.02$ & $0.67 \pm 0.13-0.05$ \\
\hline A3667 & $0.10-0.20$ & $6.32 \pm 0.10$ & $0.48 \pm 0.06+0.01$ & $0.53 \pm 0.10-0.03$ \\
\hline A3667 & $0.20-0.30$ & $6.85 \pm 0.29$ & $0.36 \pm 0.06-0.04$ & $0.39 \pm 0.09+0.02$ \\
\hline A3667 & $0.30-0.50$ & $6.33 \pm 0.26$ & $0.29 \pm 0.05+0.01$ & - \\
\hline A426 & $0.00-0.03$ & $3.79 \pm 0.01$ & $0.78 \pm 0.02+0.13$ & - \\
\hline A426 & $0.03-0.06$ & $5.39 \pm 0.04$ & $0.66 \pm 0.05+0.14$ & $0.70 \pm 0.15-0.20$ \\
\hline A426 & $0.06-0.10$ & $6.28 \pm 0.07$ & $0.54 \pm 0.06+0.06$ & $0.56 \pm 0.10-0.06$ \\
\hline A426 & $0.10-0.20$ & $6.73 \pm 0.07$ & $0.52 \pm 0.07-0.01$ & $0.40 \pm 0.05+0.01$ \\
\hline A426 & $0.20-0.30$ & $6.54 \pm 0.16$ & $0.48 \pm 0.07+0.03$ & $0.33 \pm 0.08-0.02$ \\
\hline A426 & $0.30-0.50$ & $5.58 \pm 0.24$ & $0.48 \pm 0.06+0.07$ & - \\
\hline A2256 & $0.03-0.06$ & $7.12 \pm 0.63$ & $0.55 \pm 0.15-0.06$ & - \\
\hline A2256 & $0.06-0.10$ & $6.70 \pm 0.33$ & $0.52 \pm 0.10+0.02$ & $0.86 \pm 0.21-0.05$ \\
\hline A2256 & $0.10-0.20$ & $6.77 \pm 0.30$ & $0.46 \pm 0.08-0.01$ & $0.52 \pm 0.12-0.01$ \\
\hline A2256 & $0.20-0.30$ & $8.08 \pm 0.41$ & $0.54 \pm 0.13-0.15$ & - \\
\hline A2256 & $0.30-0.50$ & $9.11 \pm 1.25$ & $0.41 \pm 0.16-0.15$ & - \\
\hline A3571 & $0.00-0.03$ & $6.66 \pm 0.21$ & $0.67 \pm 0.10-0.01$ & $0.65 \pm 0.13-0.02$ \\
\hline A3571 & $0.03-0.06$ & $6.83 \pm 0.21$ & $0.68 \pm 0.10+0.01$ & $0.84 \pm 0.14-0.06$ \\
\hline A3571 & $0.06-0.10$ & $6.90 \pm 0.19$ & $0.59 \pm 0.09-0.05$ & $0.54 \pm 0.09+0.03$ \\
\hline A3571 & $0.10-0.20$ & $6.71 \pm 0.26$ & $0.56 \pm 0.09-0.02$ & $0.39 \pm 0.09-0.01$ \\
\hline A3571 & $0.20-0.30$ & $7.82 \pm 0.88$ & $0.63 \pm 0.21-0.12$ & - \\
\hline A2029 & $0.00-0.03$ & $6.48 \pm 0.17$ & $1.08 \pm 0.16+0.19$ & $1.21 \pm 0.26-0.08$ \\
\hline A2029 & $0.03-0.06$ & $7.63 \pm 0.29$ & $0.86 \pm 0.17+0.02$ & $0.93 \pm 0.16-0.03$ \\
\hline A2029 & $0.06-0.10$ & $7.45 \pm 0.33$ & $0.65 \pm 0.13+0.05$ & $0.57 \pm 0.13-0.02$ \\
\hline A2029 & $0.10-0.20$ & $7.75 \pm 0.41$ & $0.57 \pm 0.13+0.07$ & $0.75 \pm 0.14-0.03$ \\
\hline A2029 & $0.20-0.30$ & $8.03 \pm 0.94$ & $0.44 \pm 0.17-0.05$ & - \\
\hline A1656 & $0.00-0.03$ & $8.17 \pm 0.09$ & $0.41 \pm 0.07+0.05$ & $0.46 \pm 0.03-0.02$ \\
\hline A1656 & $0.03-0.06$ & $8.22 \pm 0.06$ & $0.39 \pm 0.07-0.03$ & $0.41 \pm 0.03+0.01$ \\
\hline A1656 & $0.06-0.10$ & $8.11 \pm 0.07$ & $0.40 \pm 0.07-0.00$ & $0.39 \pm 0.03-0.00$ \\
\hline A1656 & $0.10-0.20$ & $7.75 \pm 0.05$ & $0.39 \pm 0.07-0.02$ & $0.39 \pm 0.03+0.01$ \\
\hline A1656 & $0.20-0.30$ & $6.78 \pm 0.13$ & $0.31 \pm 0.04+0.08$ & $0.31 \pm 0.07-0.04$ \\
\hline A1656 & $0.30-0.50$ & $5.40 \pm 0.20$ & $0.25 \pm 0.03+0.03$ & - \\
\hline A3266 & $0.00-0.03$ & $7.59 \pm 0.44$ & $0.60 \pm 0.14+0.06$ & $0.79 \pm 0.17-0.04$ \\
\hline A3266 & $0.03-0.06$ & $8.29 \pm 0.39$ & $0.58 \pm 0.13-0.02$ & $0.55 \pm 0.11-0.00$ \\
\hline A3266 & $0.06-0.10$ & $8.21 \pm 0.31$ & $0.49 \pm 0.10+0.00$ & $0.38 \pm 0.09-0.01$ \\
\hline A3266 & $0.10-0.20$ & $8.09 \pm 0.21$ & $0.46 \pm 0.09-0.02$ & $0.46 \pm 0.06+0.01$ \\
\hline A3266 & $0.20-0.30$ & $6.86 \pm 0.30$ & $0.33 \pm 0.07+0.03$ & $0.42 \pm 0.12-0.00$ \\
\hline A3266 & $0.30-0.50$ & $6.29 \pm 0.47$ & $0.48 \pm 0.12+0.13$ & - \\
\hline A754 & $0.03-0.06$ & $8.93 \pm 0.60$ & $0.45 \pm 0.12+0.03$ & $0.62 \pm 0.13-0.01$ \\
\hline A754 & $0.06-0.10$ & $8.85 \pm 0.42$ & $0.58 \pm 0.13-0.02$ & $0.36 \pm 0.08+0.01$ \\
\hline
\end{tabular}


Table 2. continued.

\begin{tabular}{lllll}
\hline \hline Cluster & $r\left(r_{180}\right)$ & $k T^{a}(\mathrm{keV})$ & $\begin{array}{l}\text { Fe (solar) from } F_{\mathrm{FeHeK}} / F_{3.5-6} \\
\text { Best-fit }^{b} \pm \Delta_{\text {stat }^{c} \Delta_{\text {sys }}{ }^{d}}\end{array}$ & $\begin{array}{l}\text { Fe (solar) from } F_{\mathrm{FeHK}} / F_{3.5-6} \\
\text { Best-fit }^{b} \pm \Delta_{\text {stat }^{c}{ }^{c} \Delta_{\text {sys }}{ }^{2}}\end{array}$ \\
\hline A754 & $0.10-0.20$ & $9.66 \pm 0.38$ & $0.52 \pm 0.11-0.06$ & $0.47 \pm 0.06+0.01$ \\
A754 & $0.20-0.30$ & $10.04 \pm 1.67$ & $0.38 \pm 0.16-0.04$ & $0.54 \pm 0.12-0.01$ \\
\hline
\end{tabular}

Notes. ${ }^{(a)}$ Best-fit ICM temperature with $1 \sigma$ statistical error derived from the $1 \mathrm{~T}$ model fits. ${ }^{(b)}$ Best-fit Fe abundance using the ICM temperature derived from the $1 \mathrm{~T}$ model fits. ${ }^{(c)} 1 \sigma$ statistical error, considering a $10 \%$ systematic uncertainty in the ICM temperature derived from the $1 \mathrm{~T}$ model. ${ }^{(d)}$ Difference in the derived Fe abundances using the best-fit multi-T model and the 1T model.

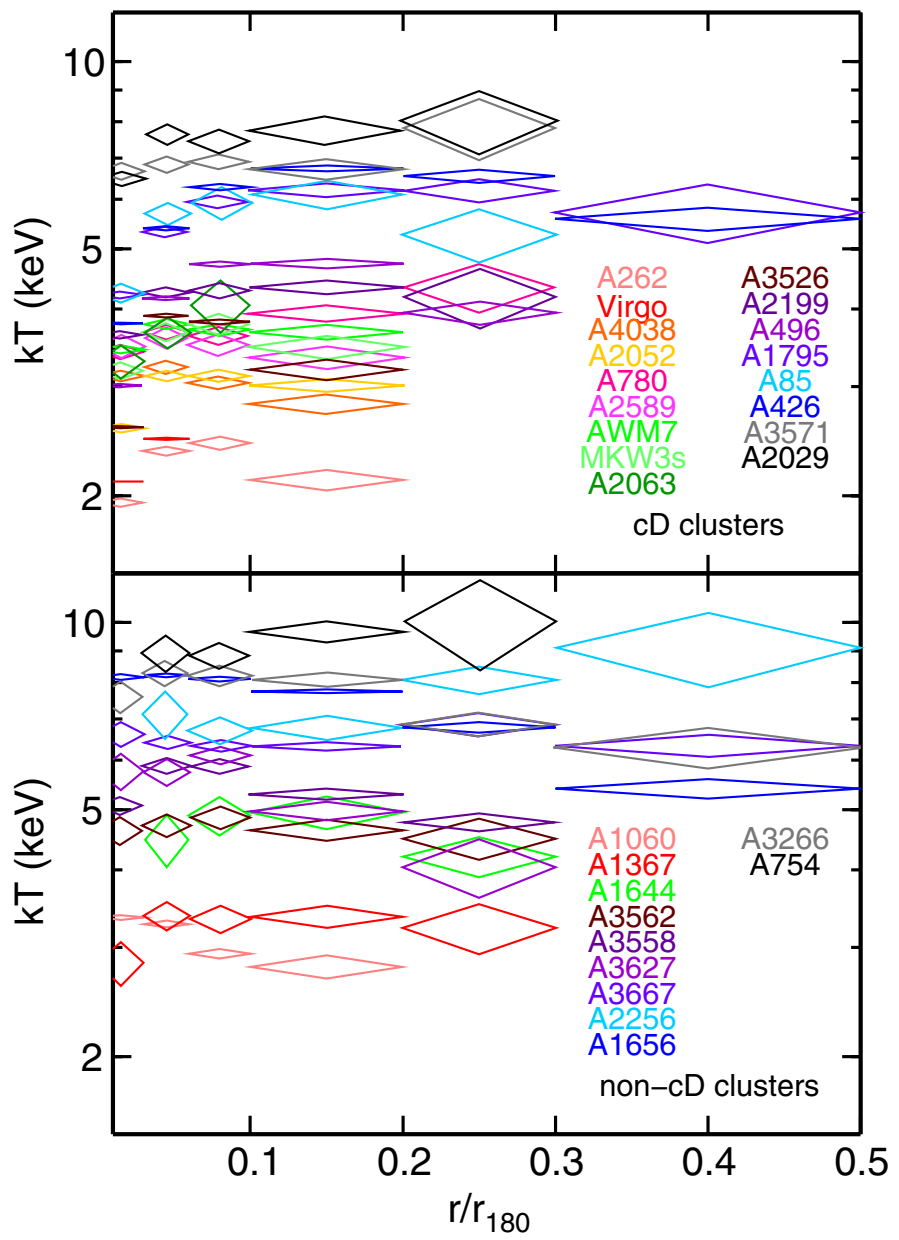

Fig. 3. Radial profiles of the ICM temperature of $\mathrm{cD}$ clusters (upper panel) and non-cD clusters (lower panel).

temperature, or between the $\mathrm{cD}$ and non-cD clusters. The scatter in the Fe abundance tends to be larger in lower temperature clusters. When the ICM temperature is higher than $5 \mathrm{keV}$, the scatter in the absolute values of the $\mathrm{Fe}$ abundances at $0.06-0.3 r_{180}$ is small.

\subsection{Systematic uncertainties in Fe abundance}

In determining the abundance, we must examine uncertainties such as the dependence on the temperature structure and plasma codes.

When the ICM temperature derived from the $1 \mathrm{~T}$ model was higher than $5 \mathrm{keV}$, the observed $F_{\mathrm{FeHK}} / F_{3.5-6}$ was converted to the Fe abundance as described in Sect. 4.2. The derived Fe abundances agree well, within statistical errors, with those derived from $F_{\mathrm{FeHeK}} / F_{3.5-6}$ (upper panel in Fig. 8, Table 2). On average, the difference between the Fe abundances derived from the two lines is only a few $\%$. At the center of the cool core, where the temperature and abundance gradients of the ICM are steep, the $\mathrm{Fe}$ abundances derived from the He-like line can be expected to be higher than those from the H-like line. However, outside the cool core, where the temperature and abundance gradients weaken, it is reasonable that He-like and $\mathrm{H}$-like lines yield the same Fe abundances. Because the temperature dependence of the He-like and H-like Fe lines are completely different, the consistency of the $\mathrm{Fe}$ abundances outside the cool cores indicates a small systematic uncertainty owing to the uncertainties in the ICM temperature.

To examine the systematic effect of the assumptions about the temperature structure, i.e., the $1 \mathrm{~T}$ model or the multi-T model, we derived the $\mathrm{Fe}$ abundance from the observed $F_{\mathrm{FeHeK}} / F_{3.5-6}$ and $F_{\mathrm{FeHK}} / F_{3.5-6}$ assuming the best-fit multi-T model. For both He-like and H-like lines, the multi-T model yields mostly the same Fe abundances as the 1T model, as summarized in Fig. 9 and Table 2. In Fig. 9, the error bars arise from a systematic uncertainty of $10 \%$ in the ICM temperature, because in most cases these errors are much larger than those from the uncertainty in the multi-T model. Statistical errors in the $\mathrm{Fe}$ line strengths are not considered here, because we use the same line strength.

As summarized in Table 3, the differences in the Fe abundances from $F_{\mathrm{FeHeK}} / F_{3.5-6}$ are smaller than several percent particularly below $5 \mathrm{keV}$ owing to the weaker temperature dependence. Above $5 \mathrm{keV}$, the systematic differences are still smaller than $\sim 10 \%$. The differences in the Fe abundances from $F_{\mathrm{FeHK}} / F_{3.5-6}$ are also about several percent owing to the weaker temperature dependence.

Figure 8 also compares the $\mathrm{Fe}$ abundances from the two lines, assuming the best-fit multi-temperature model. Here, statistical errors in the line strengths and a $10 \%$ systematic uncertainty in the temperature are considered. In most clusters, the two lines yield the same $\mathrm{Fe}$ abundances, reflecting that the $1 \mathrm{~T}$ and multi-T models yielded the same Fe abundances.

Higher-temperature components may exist in the ICM. Therefore, we left the highest temperature of the multi-T model unconstrained and fitted the spectra from the PN and MOS. For the ICM temperatures from the $1 \mathrm{~T}$ model below $5 \mathrm{keV}$ we obtained almost the same $\mathrm{Fe}$ abundances within several percent. When the ICM temperature is above $5 \mathrm{keV}$, the Fe abundances using the best-fit multi-T model from the PN spectra were unchanged. However, using the MOS multi-T model, the Fe abundances from the He-like line increased by a several tens of percent, whereas those from the $\mathrm{H}$-like line remained the same within $10 \%$. This discrepancy arises from a systematic uncertainty of several percent in the response matrices of the PN and MOS. Considering the weak temperature dependence of the $F_{\mathrm{FeHK}} / F_{3.5-6}$ at higher temperatures, the Fe abundances from the $\mathrm{H}$-like line are more reliable outside the cool core regions. 
K. Matsushita: Fe abundance in the intracluster medium

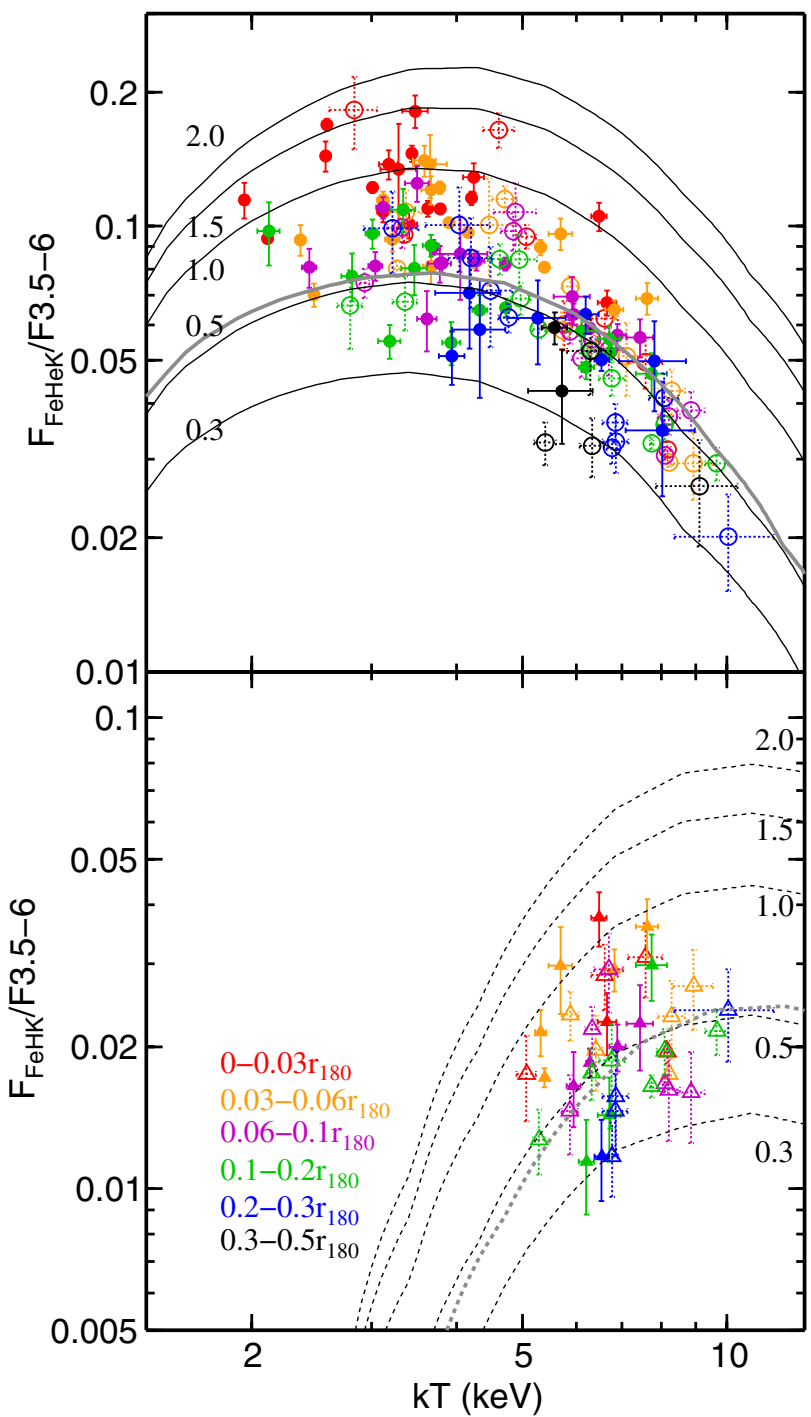

Fig. 4. $F_{\mathrm{FeHeK}} / F_{3.5-6}$ (upper panel; circles) and $F_{\mathrm{FeHK}} / F_{3.5-6}$ (lower panel; triangles) at $0-0.03 r_{180}$ (red), $0.03-0.06 r_{180}$ (orange), 0.06-0.1 $r_{180}$ (magenta) $0.1-0.2 r_{180}$ (green), $0.2-0.3 r_{180}$ (blue), and $0.3-0.5 r_{180}$ (black), plotted against the ICM temperature. Closed and open symbols indicate cD clusters and non-cD clusters, respectively. Black solid and dotted lines correspond to the theoretical ratios from the APEC model of $F_{\mathrm{FeHeK}} / F_{3.5-6}$ and $F_{\mathrm{FeHK}} / F_{3.5-6}$, respectively, assuming $\mathrm{Fe}$ abundance $=0.3,0.5,1.0,1.5$, and 2.0 solar. Here, abundance ratios of elements are assumed to have the solar ratios by Lodders (2003). Those of the MEKAL model assuming Fe abundance $=0.5$ solar are plotted as gray lines.

Rasia et al. (2008) studied bias or systematic effects in the X-ray spectra using the spectra simulated by the X-ray Map Simulator and found that the Fe abundance is recovered with high accuracy for hot $(T>3 \mathrm{keV})$ and cold $(T<2 \mathrm{keV})$ systems. At intermediate temperatures $(2<T<3 \mathrm{keV})$, they found systematic overestimates. The problem occurs because of a transition between the relative importance of the Fe-L and $\mathrm{Fe}-\mathrm{K}$ lines. Because we here derived the $\mathrm{Fe}$ abundances from the $\mathrm{Fe}-\mathrm{K}$ lines, the observed values do not include those of the coolest components (below $\sim 1.7 \mathrm{keV}$ ). The fraction of the emission measure for temperature components below this temperature is smaller than several percent except in the innermost
Table 3. Averages of the relative differences in the derived Fe abundances $\left[\left(\mathrm{Fe}_{2}-\mathrm{Fe}_{1}\right) / \mathrm{Fe}_{1}\right]$ and their root-mean-square scatters.

\begin{tabular}{ccccc}
\hline \hline $\mathrm{Fe}_{1}$ & $\mathrm{Fe}_{2}$ & Sample & Average & Scatter \\
\hline $\mathrm{FeHeK}, 1 \mathrm{~T}$ & $\mathrm{FeHeK}$, multi-T & cool & 0.01 & 0.06 \\
$\mathrm{FeHeK}, 1 \mathrm{~T}$ & $\mathrm{FeHeK}$, multi-T & hot & 0.02 & 0.10 \\
$\mathrm{FeHK}, 1 \mathrm{~T}$ & FeHK, multi-T & hot & -0.04 & 0.07 \\
\hline
\end{tabular}

Notes. Cool (hot) samples have local ICM temperatures below (above) $5 \mathrm{keV}$.

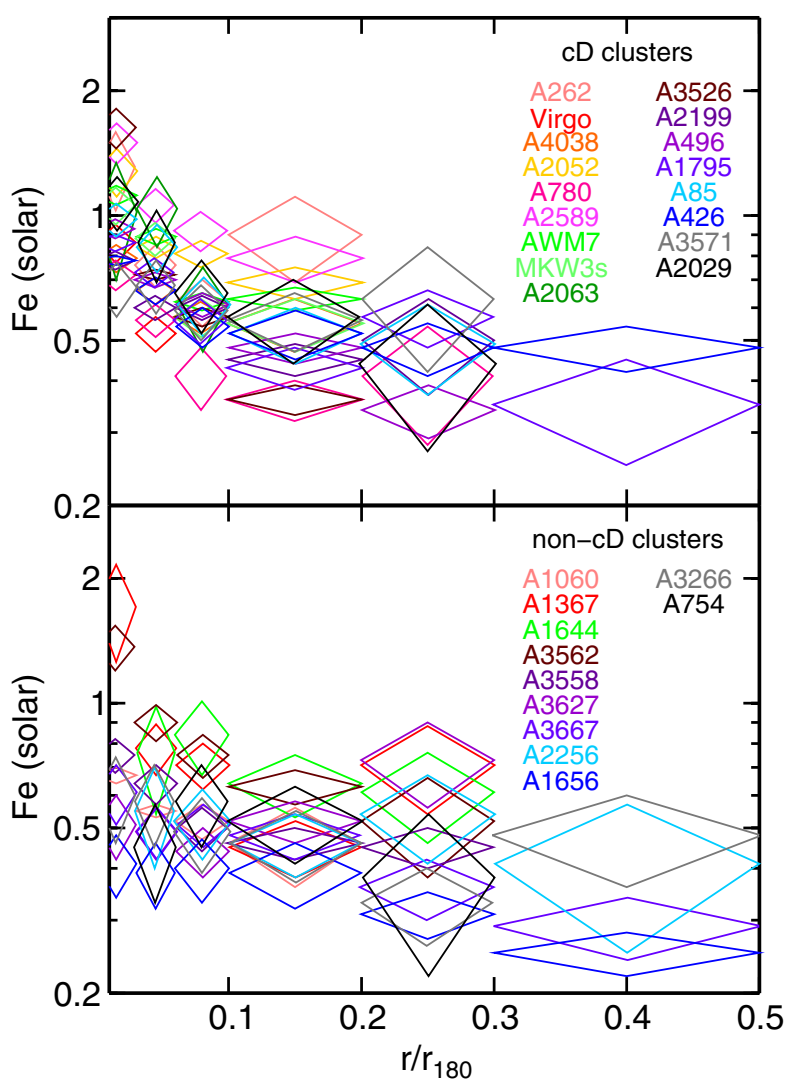

Fig. 5. Radial profiles of the Fe abundances of cD clusters (upper panel) and non-cD clusters (lower panel).

region of the coolest cool cores of the Virgo cluster and A 262, where the fractions are $30 \%$.

To examine the systematic effect of the plasma code on abundance determination, we plotted the temperature dependence of the ratio of line strength to $F_{3.5-6}$ in Fig. 4 using the MEKAL model (Mewe et al. 1995, 1996; Kaastra 1992; Liedahl et al. 1995). The difference between the MEKAL and APEC models is less than $10 \%$.

Non-solar abundance ratios of $\mathrm{He}, \mathrm{C}, \mathrm{N}$, and $\mathrm{O}$ to $\mathrm{Fe}$ cause a bias in the derived $\mathrm{Fe}$ abundance, since these elements change the continuum level. The difference in the He abundance between the solar abundance tables by Lodders (2003) and Anders \& Grevesse (1989) yields a bias of several percent. The bias resulting from a possible high $\mathrm{He}$-abundance in the ICM is discussed in detail in Ettori \& Fabian (2006) and Böhringer \& Werner (2010).

In summary, in most cases, the systematic uncertainty in the derived $\mathrm{Fe}$ abundances caused by the uncertainties in the temperature structures and atomic data is about $10 \%$. 


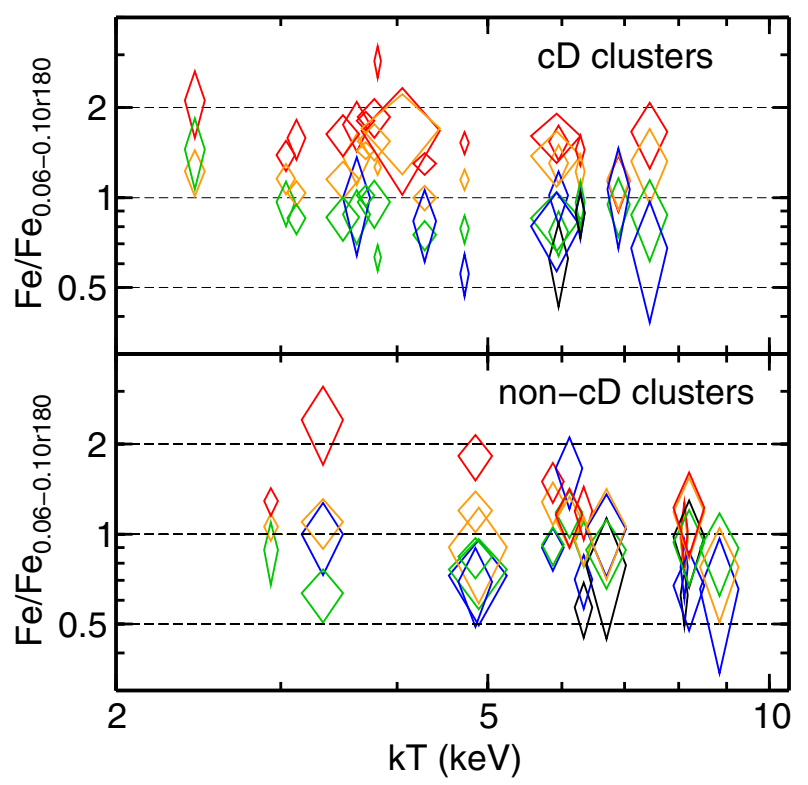

Fig. 6. Ratios of $\mathrm{Fe}$ abundances derived from $F_{\mathrm{FeHeK}} / F_{3.5-6}$ at $0-0.03 r_{180}$ (red), $0.03-0.06 r_{180}$ (orange), $0.1-0.2 r_{180}$ (green), $0.2-0.3 r_{180}$ (blue), and $0.3-0.5 r_{180}$ (black) to those at $0.06-0.1 r_{180}$, plotted against the ICM temperature at 0.06-0.1 $r_{180}$. Upper and lower panels correspond to the $\mathrm{cD}$ and non-cD clusters, respectively.

\subsection{Weighted average of Fe abundance profiles}

We calculated the weighted average of the Fe abundances derived from $F_{\mathrm{FeHeK}} / F_{3.5-6}$ and $F_{\mathrm{FeHK}} / F_{3.5-6}$, using the $1 \mathrm{~T}$ APEC model within the same radial region in the units of $r_{180}$. We divided the clusters except for A426 (the Perseus cluster) and A1656 (the Coma cluster) according to whether they are $\mathrm{cD}$ or non-cD clusters.

The results are summarized in Table 4 and Fig. 10. The radial profile of the average $\mathrm{Fe}$ abundance of the $\mathrm{cD}$ clusters becomes flatter from 0.1 to $0.3 r_{180}$ at $0.4-0.5$ solar. The Perseus cluster has a $10 \%$ smaller radial profile than the other $\mathrm{cD}$ clusters within $0.3 r_{180}$, and a flatter Fe abundance profile up to $0.5 r_{180}$. In contrast, the non-cD clusters have systematically lower Fe abundances within $0.1 r_{180}$, and the values similar to those of $\mathrm{cD}$ clusters beyond this radius. The Coma cluster also has a flatter radial profile within $0.2 r_{180}$, but at a lower value of $\sim 0.4$ solar, and beyond this radius, the $\mathrm{Fe}$ abundance gradually decreases to $\sim 0.3$ solar.

\subsection{Comparison with previous papers and redshift evolution}

In Fig. 10 we compare our results with those obtained by Leccardi \& Molendi (2008). Here, the Fe abundances were scaled using Lodders (2003), considering the difference in the solar values of $\mathrm{He}$ and Fe. Leccardi \& Molendi (2008) analyzed $\sim 50$ hot $(k T>3.3 \mathrm{keV})$ intermediate-redshift $(z=0.1 \sim$ 0.3 ) clusters of galaxies observed with XMM-Newton. Although we use samples different from those employed by Leccardi \& Molendi (2008),

our mean profiles of the cD clusters and those of Leccardi \& Molendi (2008) have remarkably similar slopes, but the absolute values differ by $10 \sim 20 \%$.

Maughan et al. (2008) analyzed redshift evolution up to $z \sim 1$ of the Fe abundance in the ICM using Chandra observations. The mean value of Maughan et al. (2008) at $0.15-1 r_{500}$ of $z<0.5$ clusters is $\sim 0.5$ solar using the solar abundance

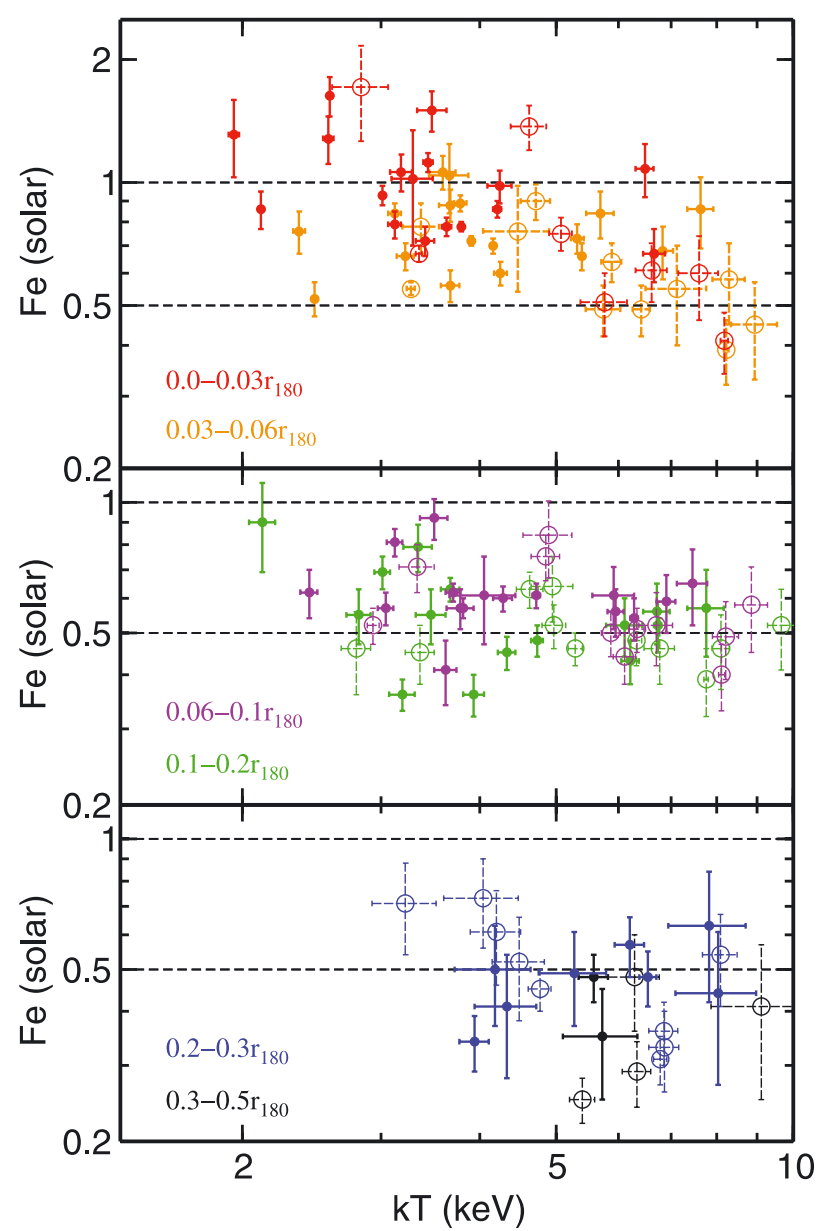

Fig. 7. Fe abundances derived from $F_{\mathrm{FeHeK}} / F_{3.5-6}$ using the 1T APEC model, plotted against the local ICM temperature. The indications of symbols and colors are the same as in Fig. 4.

by Lodders (2003), and is consistent with the average of the cD clusters.

These agreements indicate that the evolution of the Fe abundance with redshift from $z=0$ to $z=0.5$ is consistent with no evolution within our systematic uncertainties. The expected evolution according to Ettori (2005), which is about $30 \%$ from $z \sim 0.5$ to $z \sim 0$, tends to be larger than our observations.

\section{Discussion}

\subsection{Flatter radial $\mathrm{Fe}$ abundance profile beyond $0.1 r_{180}$}

Beyond $0.1 r_{180}$, well outside the cool cores, both the cD and non$\mathrm{cD}$ clusters have similar $\mathrm{Fe}$ abundance profiles with a relatively small scatter. Within $0.1-0.3 r_{180}$, the Fe abundances of the sample clusters are $0.4 \sim 0.5$ solar. There is no dependence on the ICM temperature, i.e., system richness. In other words, outside the central regions of clusters, the Fe abundance in the ICM may be universal in clusters of galaxies, and clusters of galaxies may have universal metal-enrichment histories. Beyond $0.3 r_{180}$, the Fe abundances become $0.2-0.5$ solar. These profiles smoothly connect with the Suzaku observations of the Fe distributions in the ICM beyond $0.5 r_{180}$ (Fujita et al. 2008; Tawa 2008).

The distribution of Fe abundance in the ICM has been modeled via hydrodynamic simulations (Fabjan et al. 2008; Kapferer et al. 2007). Figure 10 also compares our mean Fe abundance profiles with that derived by Fabjan et al. (2008). Beyond $0.1 r_{180}$, 

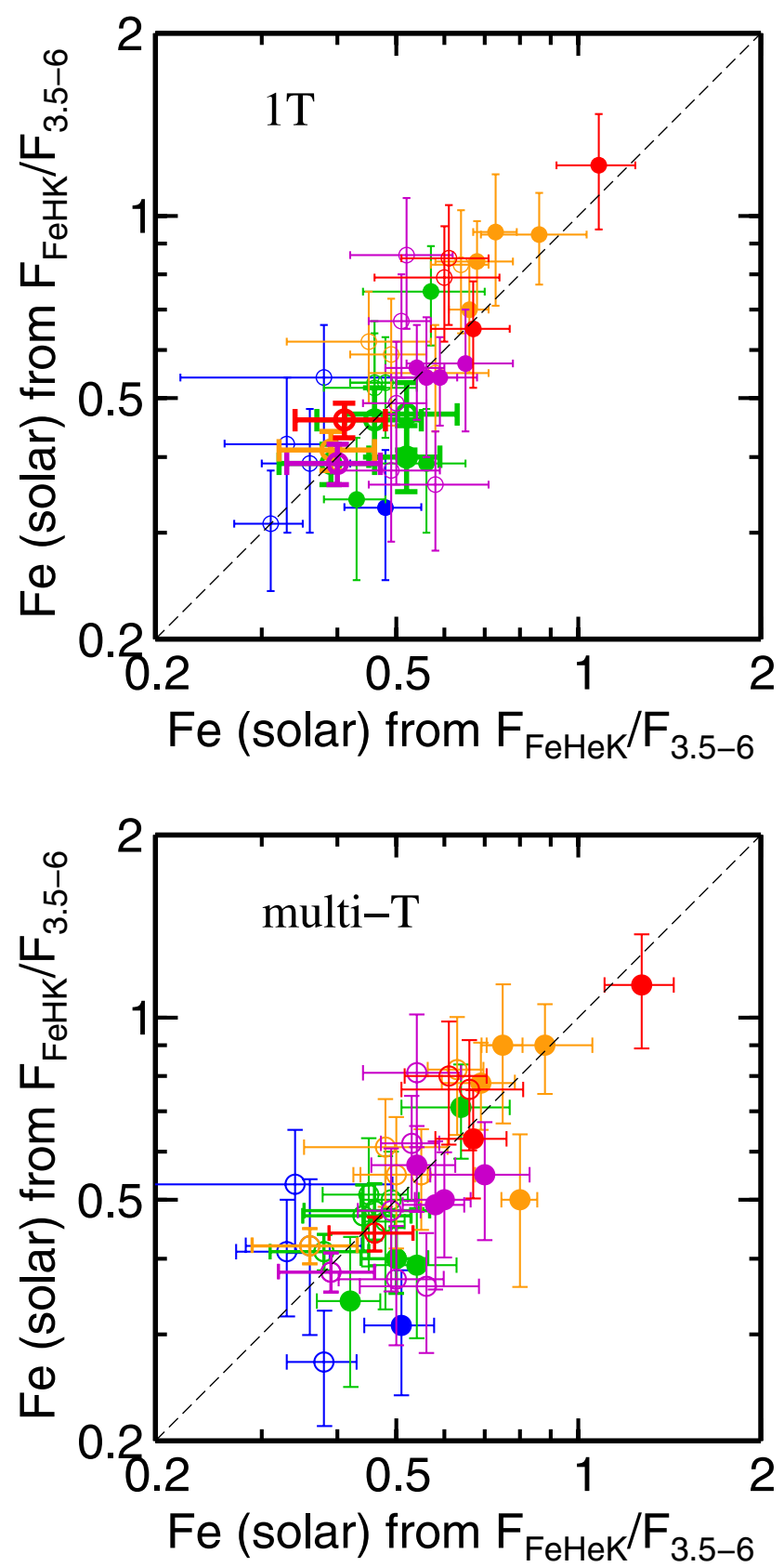

Fig. 8. Fe abundances derived from $F_{\mathrm{FeHK}} / F_{3.5-6}$ versus those from $F_{\mathrm{FeHeK}} / F_{3.5-6}$, using the $1 \mathrm{~T}$ model (upper panel) and the best-fit multi$\mathrm{T}$ model (lower panel). A systematic uncertainty of $10 \%$ in the temperature is considered. The indications of colors are the same as in Fig. 4. Closed and open circles correspond to $\mathrm{cD}$ and non-cD clusters, respectively.

the profile simulated by Fabjan et al. (2008) continues to decrease outward, whereas the observed profile becomes flatter.

The iron-mass-to-light ratio (IMLR), i.e., the ratio of $\mathrm{Fe}$ mass in the ICM to total stellar luminosity, is important for studying the origin of $\mathrm{Fe}$, because $\mathrm{Fe}$ is synthesized in stars. The integrated values of the IMLR profiles of the $k T \sim 3 \mathrm{keV}$ clusters, Abell 262, and AWM7, observed with Suzaku (Sato et al. 2008,2009 ), increase outward from $0.1 r_{180}$ to $0.3-0.4 r_{180}$. This result indicates that $\mathrm{Fe}$ in the ICM extends farther than stars in the outer regions at least in these two clusters.

A simple explanation is that a significant fraction of $\mathrm{Fe}$ is synthesized in an early phase of cluster evolution. If metal

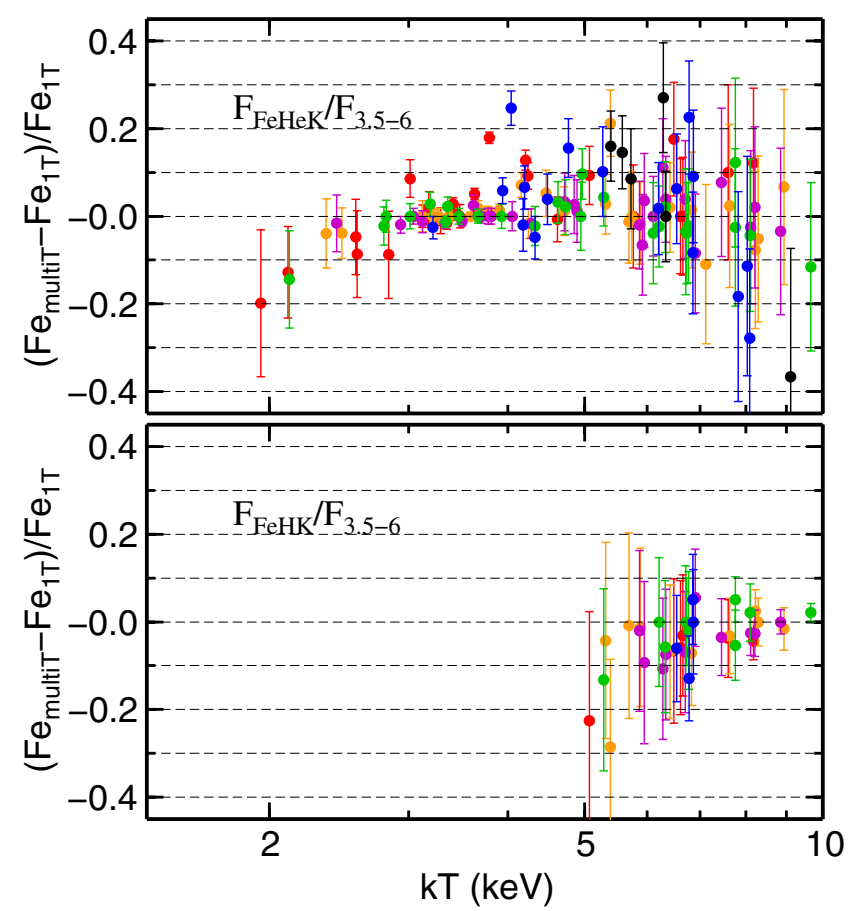

Fig. 9. Differences between the derived $\mathrm{Fe}$ abundances using the best-fit multi-T model and those using the 1T model, plotted against the ICM temperature. Error bars are the result of uncertainties in the ICM temperature derived from the $1 \mathrm{~T}$ model, including systematic uncertainties of $10 \%$. Upper and lower panels correspond to $\mathrm{Fe}$ abundances derived from $F_{\mathrm{FeHeK}} / F_{3.5-6}$ and $F_{\mathrm{FeHK}} / F_{3.5-6}$, respectively. The indications of the colors are the same as in Fig. 4.

Table 4. Weighted average of $\mathrm{Fe}$ abundances derived from $F_{\mathrm{FeHeK}} / F_{3.5-6}$ and $F_{\mathrm{FeHK}} / F_{3.5-6}$, in the units of the solar abundance.

\begin{tabular}{lcccc}
\hline \hline Radius $^{a}$ & $\mathrm{cDs}^{b}$ & $\mathrm{~A} 426$ & Non-cDs & $\mathrm{A} 1656$ \\
\hline $0.00-0.03$ & $0.88 \pm 0.02$ & $0.78 \pm 0.01$ & $0.69 \pm 0.02$ & $0.45 \pm 0.03$ \\
$0.03-0.06$ & $0.73 \pm 0.01$ & $0.66 \pm 0.05$ & $0.57 \pm 0.02$ & $0.41 \pm 0.03$ \\
$0.06-0.10$ & $0.60 \pm 0.01$ & $0.55 \pm 0.05$ & $0.52 \pm 0.02$ & $0.39 \pm 0.03$ \\
$0.10-0.20$ & $0.48 \pm 0.01$ & $0.44 \pm 0.04$ & $0.50 \pm 0.02$ & $0.39 \pm 0.03$ \\
$0.20-0.30$ & $0.42 \pm 0.04$ & $0.41 \pm 0.05$ & $0.44 \pm 0.03$ & $0.31 \pm 0.03$ \\
$0.30-0.50$ & $0.35 \pm 0.10$ & $0.48 \pm 0.06$ & $0.32 \pm 0.04$ & $0.25 \pm 0.03$ \\
\hline
\end{tabular}

Notes. ${ }^{(a)}$ In unit of $r_{180} \cdot{ }^{(b)}$ The cD clusters except A 426. ${ }^{(c)}$ The non-cD clusters except A 1656.

enrichment occurs after the formation of the clusters, the metal distribution is expected to follow the stellar distribution, whereas if it occurs before cluster formation, we expect a flatter distribution. For example, simulations by Kapferer et al. (2007) yielded a flatter Fe profile from SN II. That the measurements of the $\mathrm{Fe}$ abundance excluding the central region by Maughan et al. (2008) are consistent with no evolution at least up to $z=0.7$ also supports the early synthesis of Fe. Feedback by active galactic nuclei (AGN) may also change the abundance profile. With AGN feedback, a flatter radial profile beyond $0.1 r_{180}$ is also obtained in the simulation by Fabjan et al. (2010).

A426 (the Perseus cluster) shows a completely flat Fe profile from $0.1 r_{180}$ to $0.5 r_{180}$ : at $0.3-0.5 r_{180}$, the Fe abundance is higher than in the other clusters. Within the annular region of $0.3-0.5 r_{180}$, the observed fields of view do not cover the entire annular region (Fig. 1) and lack the north and south regions. Several clusters, including A426, form the Perseus supercluster, 
A\&A 527, A134 (2011)

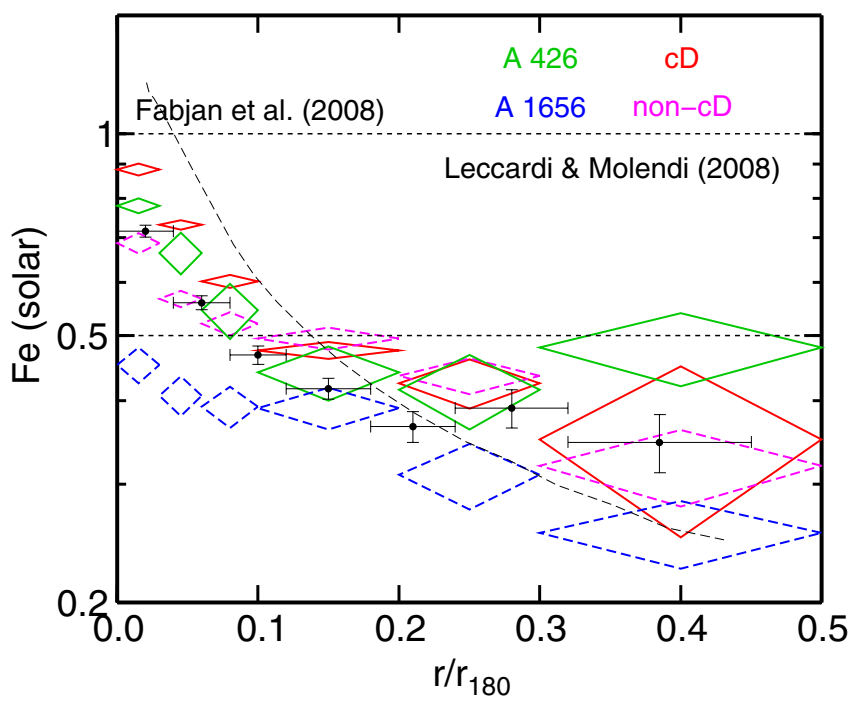

Fig. 10. Fe abundance profiles of A426 (green), A1656 (blue), and the averages of those of $\mathrm{cD}$ clusters (red solid diamonds) and non-cD clusters (magenta dashed diamonds) in our sample, compared with the average profile of $z=0.1 \sim 0.3$ clusters (black closed circles) from Leccardi \& Molendi (2008). Black dashed line corresponds to the $\mathrm{Fe}$ abundance derived from the numerical simulations without AGN feedback by Fabjan et al. (2008).

which is elongated in an east-west direction (Gregory et al. 1981). The observed very flat Fe abundance may reflect the history of cluster formation from filaments of the large-scale structure of the Universe.

\subsection{Fe profiles within the cool core regions}

$\mathrm{Fe}$ in the gas in the cool core of clusters is a mixture of that present in the ICM and later supplies from their $\mathrm{cD}$ galaxies. The latter contains Fe synthesized by SN Ia and originating from stars through stellar mass loss, because $\mathrm{Fe}$ is synthesized by both SN Ia and SN II. According to abundance patterns from XMM-Newton observations of the cool cores (Matsushita et al. 2003, 2007; Werner et al. 2006; de Plaa et al. 2006; Simionescu et al. 2008, 2009) and Suzaku observations of the cD galaxies of AWM7 and A262 (Sato et al. 2008, 2009), the number ratio of SNe II to SNe Ia is estimated to be $3 \sim 4$, and the Fe in the cluster core was synthesized mostly by SN Ia.

Within cool core regions, clusters have negative abundance gradients (e.g. Fukazawa et al. 2000; De Grandi et al. 2004). Rossetti \& Molendi (2010) and Leccardi et al. (2010) also found central metal abundance excess in low-entropy core systems. Within these regions, observations indicate that metals ejected from $\mathrm{cD}$ galaxies have a more extensive distribution compared with stars. Analyzing eight cool-core clusters observed with XMM, David \& Nulsen (2008) found that Fe in the ICM is more extended than the stellar mass, and excess Fe within the central $100 \mathrm{kpc}$ could have been produced by type Ia supernova in $\mathrm{cD}$ galaxies over the past 3-7 Gyr. The IMLR values in AWM7 and A262 observed with Suzaku have much steeper positive gradients within the cool cores than those beyond $0.1 r_{180}$ (Sato et al. 2008, 2009).

Processes such as jets from a central AGN as described below, or the "sloshing" of cD galaxies in the cluster's gravitational potential (e.g. Markevitch et al. 2001), may eject metals from cD galaxies. On the basis of 2D temperature and metallicity maps of
M 87 (Simionescu et al. 2008) and Hydra A (Simionescu et al. 2009), uplift of Fe by the AGN was discussed in detail. David \& Nulsen (2008) found that both turbulent diffusion of entropy and dissipation are important heating mechanisms in cluster cores. Rebusco et al. (2006) compared the observed Fe abundance profiles with the predictions of a model involving metal ejection from the brightest galaxy and the subsequent diffusion of metals by stochastic gas motions. Roediger et al. (2007) studied the effect of bubble-induced motions on metallicity profiles through 3D hydro simulations. The new simulation by Fabjan et al. (2010) showed a less steep central Fe peak including feedback from $\mathrm{AGN}$ in $\mathrm{cD}$ galaxies. The observed small scatter in the Fe abundance slope within $0.1 r_{180}$ in the cD clusters suggests a common process of metal supply and ejection in the cD galaxies. The next Japanese X-ray satellite, ASTRO-H, will directly reveal line broadening caused by turbulence in the cores of clusters.

Peng \& Nagai (2009) pointed out that the $\mathrm{He} / \mathrm{H}$ abundance ratio could be four times the solar abundance at the center of massive clusters. Such a high He abundance causes an underestimation of the Fe abundance by a factor of two (Ettori \& Fabian 2006). However, in actual clusters, metals at the center of the cluster come from mass loss by central galaxies, and in addition, more extensively distributed compared with stars. Therefore, the centers of clusters should not be static, as assumed in the calculations of Peng \& Nagai (2009).

\subsection{Mixing of the ICM in non-cD clusters}

Most of clusters without cool cores tend to show no central abundance excess as found in Fukazawa et al. (2000); De Grandi et al. (2004). Leccardi et al. (2010) found that some medium- and high-entropy core systems have central abundance excess, most of which are undergoing a phase of rapid dynamical changes, and suggested that these clusters originate from low-entropy core systems.

Our results are consistent with the discussion in Leccardi et al. (2010). The non-cD clusters in this paper include merging clusters in various stages, from heavily distorted morphologies to relatively relaxed ones without cool cores. During cluster merging, mixing of the ICM could destroy the central Fe peak. Clusters with the highest central Fe abundances have highly disturbed morphologies. In the first stage of merging, the Fe peak and the cool core may remain. Then, mixing of the ICM destroys the Fe peak, and the Fe abundance becomes flat at the same level as in $\mathrm{cD}$ clusters at $0.1-0.5 r_{180}$.

\section{Summary and conclusion}

We derived radial profiles of the Fe abundance of the ICM in nearby $(z<0.08)$ clusters observed with XMM-Newton. The Fe abundances of the ICM were derived from the ratio of the flux of the $\mathrm{K} \alpha$ lines of He-like or H-like Fe to those of the continuum at $3.5-6.0 \mathrm{keV}$, because the systematic uncertainty in the continuum flux in this energy band owing to the background is smaller. The temperature dependence of these ratios constrains the Fe abundances of multi-temperature plasmas.

In cluster core regions $\left(<0.1 r_{180}\right)$, the observed $\mathrm{Fe}$ abundances of $\mathrm{cD}$ clusters show similar radial profiles. The lesspeaked abundance profile compared with the light in the central region indicates the ejection of metals from $\mathrm{cD}$ galaxies.

In the outer regions, $0.1-0.2 r_{180}, \mathrm{Fe}$ abundances of $0.4-0.5$ solar appear to be universal with no temperature dependence. 
The observed flatter Fe abundance profiles of the $\mathrm{cD}$ clusters beyond $0.1 r_{180}$ indicate early metal enrichment.

Chandra and XMM cannot reach beyond $0.5 r_{180}$ owing to a high, unstable particle background. Information is available for only about $10 \%$ of the cluster volume, and the majority has not yet been revealed. The total amount of Fe synthesized in galaxies can be derived only by the precise abundance measurements beyond $0.5 r_{180}$. Because of its low background, Suzaku is the only satellite available for the next several years to study clusters of galaxies up to the virial radius. With Suzaku, Fe abundances will be determined out to a radius of $0.7 \sim 0.8 r_{180}$.

\section{References}

Anders, E., \& Grevesse, N. 1989, Geochim. Cosmochim. Acta, 53, 197 Arnaud, M., Rothenflug, R., Boulade, O., Vigroux, L., \& Vangioni-Flam, E. 1992, A\&A, 254, 49

Baldi, A., Ettori, S., Mazzotta, P., Tozzi, P., \& Borgani, S. 2007, ApJ, 666, 835 Böhringer, H., \& Werner, N. 2010, A\&ARv, 18, 127

Böhringer, H., Matsushita, K., Churazov, E., et al. 2002, A\&A, 382, 804

David, L. P., \& Nulsen, P. E. J. 2008, ApJ, 689, 837

De Grandi, S., Ettori, S., Longhetti, M., \& Molendi, S. 2004, A\&A, 419, 7

de Plaa, J., Werner, N., Bykov, A. M., et al. 2006, A\&A, 452, 397

Dickey, J. M., \& Lockman, F. J. 1990, ARA\&A, 28, 215

Ebeling, H., Voges, W., Bohringer, H., et al. 1996, MNRAS, 281, 799

Ettori, S. 2005, MNRAS, 362, 110

Ettori, S., \& Fabian, A. C. 2006, MNRAS, 369, L42

Ezawa, H., Fukazawa, Y., Makishima, K., et al. 1997, ApJ, 490, L33

Ezawa, H., Yamasaki, N. Y., Ohashi, T., et al. 2001, PASJ, 53, 595

Fabjan, D., Tornatore, L., Borgani, S., Saro, A., \& Dolag, K. 2008, MNRAS, 386,1265

Fabjan, D., Borgani, S., Tornatore, L., et al. 2010, MNRAS, 401, 1670

Finoguenov, A., David, L. P., \& Ponman, T. J. 2000, ApJ, 544, 188

Finoguenov, A., Arnaud, M., \& David, L. P. 2001, ApJ, 555, 191

Fujita, Y., Tawa, N., Hayashida, K., et al. 2008, PASJ, 60, 343

Fukazawa, Y., Makishima, K., Tamura, T., et al. 1998, PASJ, 50, 187

Fukazawa, Y., Makishima, K., Tamura, T., et al. 2000, MNRAS, 313, 21

Gregory, S. A., Thompson, L. A., \& Tifft, W. G. 1981, ApJ, 243, 411

Kaastra, J. S. 1992, An X-Ray Spectral Code for Optically Thin Plasmas (Internal SRON-Leiden Report, updated version 2.0)

Katayama, H., Takahashi, I., Ikebe, Y., Matsushita, K., \& Freyberg, M. 2004, A\&A, 414, 767
Kapferer, W., Kronberger, T., Weratschnig, J., et al. 2007, A\&A, 466, 813 Leccardi, A., \& Molendi, S. 2008, A\&A, 487, 461

Leccardi, A., Rossetti, M., \& Molendi, S. 2010, A\&A, 510, A82

Liedahl, D. A., Osterheld, A. L., \& Goldstein, W. H. 1995, ApJ, 438, L115

Lodders, K. 2003, ApJ, 591, 1220

Lumb, D. H., Warwick, R. S., Page, M., \& De Luca, A. 2002, A\&A, 389, 93

Matsushita, K., Finoguenov, A., \& Böhringer, H. 2003, A\&A, 401, 443

Matsushita, K., Böhringer, H., Takahashi, I., \& Ikebe, Y. 2007, A\&A, 462, 953

Mewe, R., Gronenschild, E. H. B. M., \& van den Oord, G. H. J. 1985, A\&AS, 62, 197

Mewe, R., Lemen, J. R., \& van den Oord, G. H. J. 1986, A\&AS, 65, 511

Makishima, K., Ezawa, H., Fukuzawa, Y., et al. 2001, PASJ, 53, 401

Markevitch, M., Forman, W. R., Sarazin, C. L., \& Vikhlinin, A. 1998, ApJ, 503, 77

Markevitch, M., Vikhlinin, A., \& Mazzotta, P. 2001, ApJ, 562, L153

Maughan, B. J., Jones, C., Forman, W., \& Van Speybroeck, L. 2008, ApJS, 174, 117

Peng, F., \& Nagai, D. 2009, ApJ, 693, 839

Rasia, E., Mazzotta, P., Bourdin, H., et al. 2008, ApJ, 674, 728

Rebusco, P., Churazov, E., Böhringer, H., \& Forman, W. 2006, MNRAS, 372, 1840

Reese, E. D., Kawahara, H., Kitayama, T., et al. 2010, ApJ, 721, 653

Renzini, A., Ciotti, L., D’Ercole, A., \& Pellegrini, S. 1993, ApJ, 419, 52

Roediger, E., Brüggen, M., Rebusco, P., Böhringer, H., \& Churazov, E. 2007, MNRAS, 375,15

Rossetti, M., \& Molendi, S. 2010, A\&A, 510, A83

Sato, K., Matsushita, K., Ishisaki, Y., et al. 2008, PASJ, 60, 333

Sato, K., Matsushita, K., \& Gastaldello, F. 2009, PASJ, 61, 365

Simionescu, A., Werner, N., Finoguenov, A., Böhringer, H., \& Brüggen, M. 2008, A\&A, 482, 97

Simionescu, A., Werner, N., Böhringer, H., et al. 2009, A\&A, 493, 409

Smith, R. K., Brickhouse, N. S., Liedahl, D. A., \& Raymond, J. S. 2001, ApJ, 556,91

Snowden S. L., Mushotzky, R. F., Kuntz, K. D., \& Davis, D. S. 2008, A\&A, 478, 615

Tanaka, Y., Inoue, H., \& Holt, S. S. 1994, PASJ, 46, L37

Tamura, T., Kaastra, J. S., den Herder, J. W. A., Bleeker, J. A. M., \& Peterson, J. R. 2004, A\&A, 420, 135

Tawa, N. 2008, PhD Thesis, University of Osaka

Vikhlinin, A., Markevitch, M., Murray, S. S., et al. 2005, ApJ, 628, 655

Werner, N., de Plaa, J., Kaastra, J. S., et al. 2006, A\&A, 449, 475

Yoshino, T., Mitsuda, K., Yamasaki, N. Y., et al. 2009, PASJ, 61, 805 W

Zhang, Y.-Y., Reiprich, T. H., Finoguenov, A., Hudson, D. S., \& Sarazin, C. L. 2009, ApJ, 699, 1178 